\title{
La necrópolis calcolítica de Los Millares: dataciones radiocarbónicas y valoración de la dieta y del medio ambiente a partir del análisis de isótopos estables*
}

\author{
The Chalcolithic necropolis of Los Millares: Radiocarbon dating and an assessment of the \\ diet and the environment from stable isotope analysis
}

\author{
Fernando Molina ${ }^{\mathrm{a}}$, Alfredo Mederos ${ }^{\mathrm{b}}$, Antonio Delgado-Huertas ${ }^{\mathrm{c}}$, Juan Antonio \\ Cámara $^{a}$, Victoria Peña ${ }^{d}$, Rafael M. Martínez ${ }^{a}$, Francisco Javier Esquivel ${ }^{\mathrm{e}}$, Arsenio \\ Granados $^{c}$, Sylvia Jiménez-Brobeil ${ }^{\mathrm{f}}$ y Jose Antonio Esquivel ${ }^{\mathrm{a}}$
}

\begin{abstract}
RESUMEN
Se presentan en este estudio los resultados de los análisis isotópicos realizados sobre una muestra de restos humanos de la necrópolis calcolítica de Los Millares. Para proceder a su discusión se ha realizado previamente su contextualización cronológica a partir de una serie de dataciones radiocarbónicas y la revisión de la cronología relativa de los sepulcros. Las dataciones muestran el uso de la necrópolis megalítica durante todo el periodo de ocupación de Los Millares, al menos desde 3100/3000 hasta 2200/2100 cal AC. Los resultados del análisis isotópico apoyan las propuestas sobre un incremento en la aridez desde el 2400/2300 cal AC. Además, en una dieta dominada por los recursos terrestres en la que las proteínas suelen proceder del consumo de herbívoros, existe una cierta variabilidad con individuos que pueden haber consumido otros recursos.
\end{abstract}

\begin{abstract}
The results of the isotopic analyses performed on human remains from the Chalcolithic necropolis of Los Millares are presented in this paper. These are contextualized chronologically by a series of radiocarbon dates and, a review of the graves' relative chronology. The dates show the megalithic necropolis was in use all along the occupation period of Los Millares, at least from 3100/3000 to 2200/2100 cal BC. The isotopic analysis results support the proposals for increasing aridity from 2400/2300 cal BC onwards. Diet is mainly based on land resources, proteins being usually taken from herbivores. However, a certain variability is found among sampled individuals because some of them may have consumed others products.
\end{abstract}

Palabras clave: Calcolítico; Los Millares; Sureste de la península ibérica; Análisis isotópico; C14; Dieta; Paleoambiente.

\footnotetext{
* Este trabajo se ha realizado en el marco de los proyectos: "Dieta y movilidad en la Prehistoria Reciente de Andalucía. Un estudio de la jerarquización social a partir del registro funerario (P12-HUM-1510, Consejería de Economía, Innovación, Ciencia y Empleo de la Junta de Andalucía) y "Estrategias agropecuarias y consumo en la Edad del Bronce del sur de la península ibérica. Análisis de plantas, animales y restos humanos" (HAR201680057-P, Ministerio de Economía y Competitividad).

a Dpto. Prehistoria y Arqueología. Universidad de Granada. Facultad de Filosofía y Letras. Campus Universitario Cartuja s/n. 18071 Granada. Correos e.: FM molinag@ugr.es https://orcid.org/0000-0002-9189-4198; JAC jacamara@ugr.es https://orcid.org/0000-0003-4007-0639; RMM rmmartinez@ugr.es https://orcid.org/0000-0002-8947-117X; JAE esquivel@ugr.es https://orcid.org/0000-0002-4525-7489

b Dpto. Prehistoria y Arqueología. Facultad de Filosofía y Letras. Universidad Autónoma de Madrid. Campus de Cantoblanco. 28049 Madrid. Correo e.: alfredo.mederos@uam.es https://orcid.org/0000-0002-0036-7940

' Instituto Andaluz de Ciencias de la Tierra (CSIC-Universidad de Granada). Avenida de las Palmeras 4. 18100 Armilla. Granada. Correos e.: ADH antonio.delgado@csic.es https://orcid.org/0000-0002-7240-1570; AG arseniog@iact.ugr-csic.es https://orcid.org/0000-0002-3147-3471

d Programa de Doctorado. Universidad Complutense de Madrid. Correo e.: victoriatanit@yahoo.es https://orcid.org/0000-0001-5084-0401

e Dpto. Estadística e Investigación Operativa. Universidad de Granada. Avda de Fuente Nueva s/n. 18071 Granada. Correo e.: jesquivel@ugr.es https://orcid.org/0000-0001-8296-1013

${ }^{\mathrm{f}}$ Laboratorio de Antropología. Facultad de Medicina. Universidad de Granada. Parque Tecnológico de Ciencias de la Salud. 18071 Granada. Correo e.: jbrobeil@ugr.es https://orcid.org/0000-0001-8758-5635

Recibido 26-XII-2019; aceptado 12-II-2020.
}

Copyright: (C) 2020 CSIC. Este es un artículo de acceso abierto distribuido bajo los términos de la licencia de uso y distribución "Creative Commons Reconocimiento 4.0 Internacional” (CC BY 4.0) 
Key words: Chalcolithic; Los Millares; Southeast Iberian peninsula; Isotopic Analysis; C14; Diet; Paleoenvironment.

\section{INTRODUCCIÓN}

El yacimiento arqueológico de Los Millares fue descubierto a finales del siglo XIX (Siret 1893), junto a la población de Santa Fe de Mondújar (Almería), con motivo de la construcción de un ferrocarril minero. Desde entonces ha sido un referente en la investigación de la Prehistoria Reciente del Mediterráneo Occidental.

El conjunto calcolítico de Los Millares (Fig. 1) incluye un poblado de más de 5 ha, una necrópolis aneja de aproximadamente un centenar de sepulturas colectivas (actualmente son visibles 83) y 13 fortines que defienden el conjunto al sur y este (Molina y Cámara 2005). El asentamiento y la necrópolis se sitúan en un espolón amesetado en la confluencia del río Andarax con la Rambla de Huéchar. El primero desemboca en el golfo de Almería. El área de influencia directa de Los Millares, sin embargo, incluye también pequeños poblados y necrópolis situados en las cercanas sierras de Alhama y Gádor e incluso asentamientos y necrópolis distribuidos por las cuencas de los ríos Andarax y Nacimiento y el Cabo de Gata (Molina y Cámara 2005; Haro et al. 2006; Cámara et al.

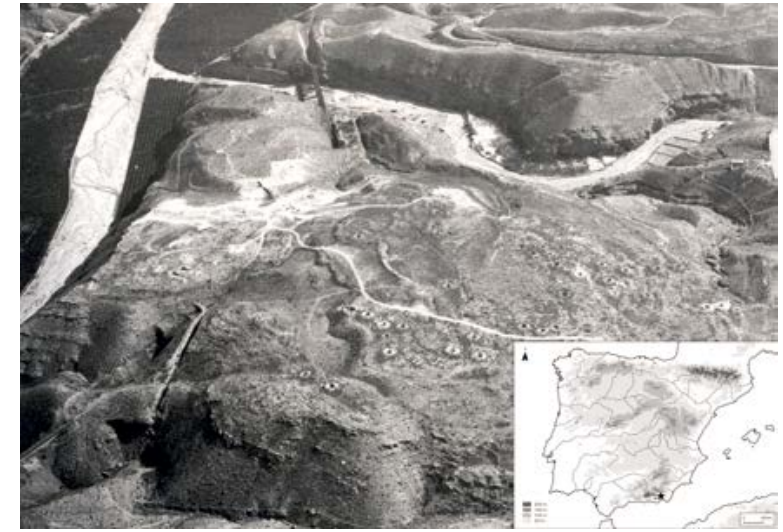

Fig. 1a. Situación del yacimiento calcolítico de Los Millares en el sureste de la Península Ibérica y vista área de su necrópolis y poblado tomada en 1979 (fotografía Altair/Grupo de Estudios de Prehistoria Reciente, GEPRAN, Universidad de Granada).

2014). Por último, en la zona más meridional de la necrópolis, junto a las tumbas de la época del Cobre, aparecen otras de arquitectura más sencilla (cistas cuadradas y rectangulares con urnas de incineración en su interior). Corresponden a una necrópolis del Bronce Final que se superpuso espacialmente a la necrópolis calcolítica, pero está desvinculada de ella como muestran la tipología de la tumbas y contenedores cerámi-

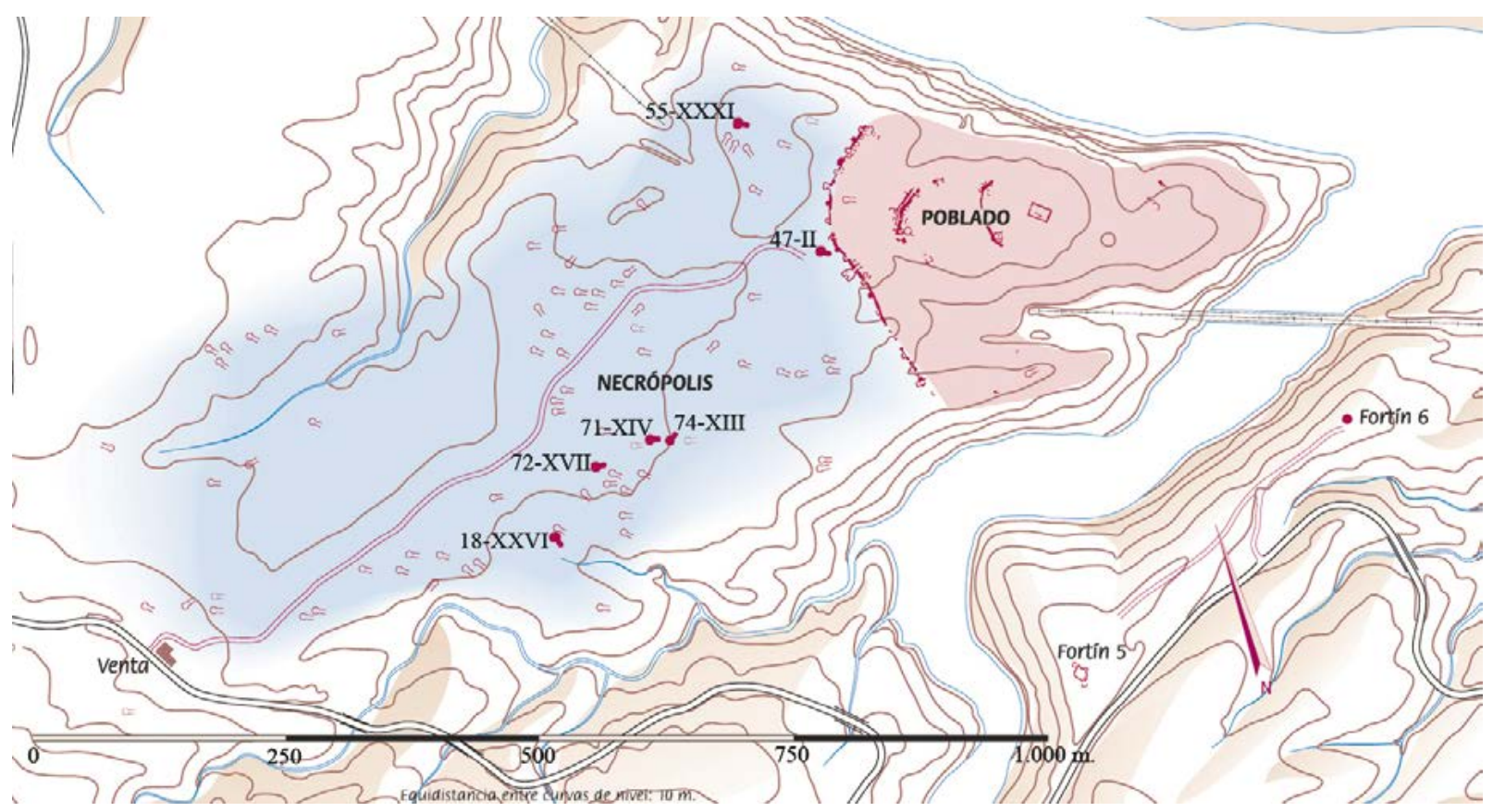

Fig. 1b. Plano simplificado de la necrópolis, con las tumbas muestreadas que aparecen numeradas, indicando con dígitos árabes la denominación de L. Siret y con dígitos romanos la de Almagro y Arribas. No se conoce la situación sobre el terreno del resto de las sepulturas muestreadas (Grupo de Estudios de Prehistoria Reciente, GEPRAN, Universidad de Granada; en color en la versión electrónica). 
cos, los ajuares funerarios y el ritual (Molina, tesis inédita ${ }^{1}$; Lorrio 2008).

Louis Siret con la ayuda de su capataz Pedro Flores llevó a cabo las primeras excavaciones, básicamente en la necrópolis, que publicó de forma no exhaustiva (Siret 1893, 1913). Con posteridad, Georg y Vera Leisner (1943) usaron la documentación de Siret para su gran corpus sobre el Megalitismo del sur de la península ibérica. En los 1950, Martín Almagro y Antonio Arribas (1963) reexcavaron unas 40 tumbas de la necrópolis, publicando solo 21 .

Las nuevas investigaciones en Los Millares por parte del equipo de la Universidad de Granada, dirigido por A. Arribas y F. Molina (Arribas et al. 1979), consistieron en actividades muy limitadas de documentación, limpieza y conservación en la necrópolis (Fig. 2) y excavaciones en el hábitat, especialmente en los sistemas de fortificación (Arribas et al. 1987).

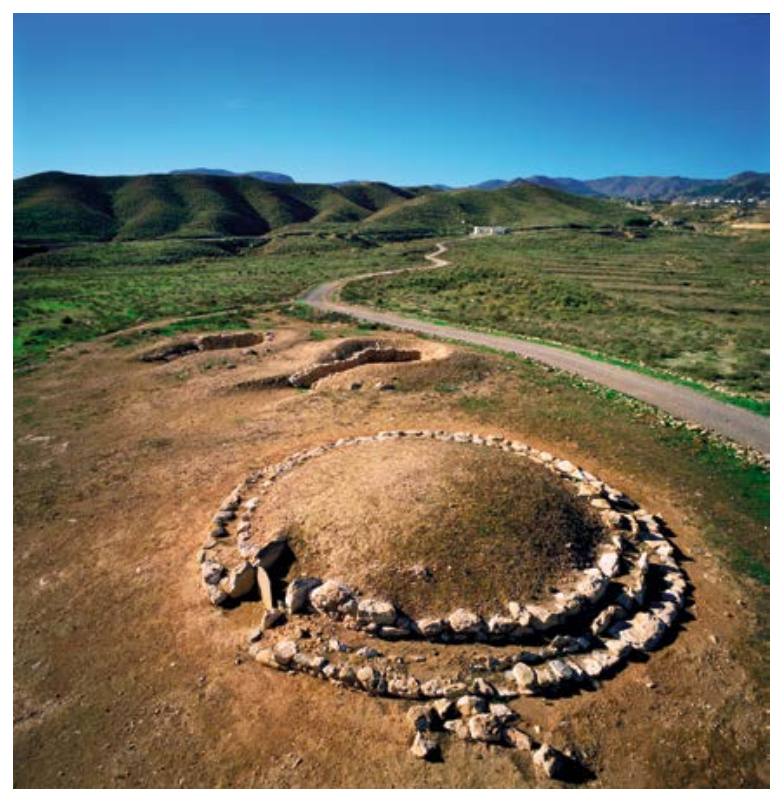

Fig. 2. Sepulturas LII a LIV de la necrópolis de Los Millares (fotografía M.A. Blanco/Consejería de Cultura de la Junta de Andalucía/Grupo de Estudios de Prehistoria Reciente, GEPRAN, Universidad de Granada; en color en la versión electrónica).

Los recintos amurallados, necrópolis y fortines que integran el sitio, dados a conocer tras las intervenciones realizadas entre finales del siglo XIX y finales del siglo XX, han sido referentes en toda discusión sobre

\footnotetext{
1 Molina González, F. 1976: La Cultura del Bronce Final en el Sudeste de la Península Ibérica. Tesis doctoral. Universidad de Granada.
}

las causas y el grado de jerarquización alcanzado por las sociedades del III milenio a. C. en el sur de la península ibérica (Gilman 1976, Chapman 1978, 1990; Hernando 1987-1988; Micó 1991; Mederos 1993; Molina y Cámara 2005; Cámara y Molina 2006; Afonso et al. 2011).

\section{OBJETIVOS E HIPÓTESIS}

Este trabajo estudia la dieta humana en el asentamiento de Los Millares y, de forma complementaria, los cambios ambientales que tuvieron lugar en el sureste de la península ibérica durante el Calcolítico, a partir del análisis de isótopos estables. Las muestras fechadas procedentes del registro funerario de su necrópolis nos han servido para evaluar posibles diferencias en la dieta entre los individuos inhumados que compartieron una misma sepultura, así como los cambios temporales en la dieta y el medioambiente.

Además, el conjunto de nuevas dataciones de C14 amplía considerablemente la información sobre la duración del uso de la necrópolis de Los Millares, ofreciendo un marco de contrastación con la cronología relativa obtenida de la tipología artefactual de los ajuares de las tumbas analizadas, fundamentalmente a partir de G. y V. Leisner (1943).

Las hipótesis relacionadas con estos objetivos son:

a) Durante la ocupación de Los Millares hubo un relativo aumento de la humedad en los siglos centrales del III milenio a. C., sugerido por los resultados de los estudios polínicos, arqueomagnéticos e isotópicos para otras áreas del sureste de la península ibérica y de la Alta Andalucía (Carrión et al. 2001, 2007; Nachasova et al. 2007; Yanes et al. 2011, 2013) y por los estudios antracológicos en el propio yacimiento (Rodríguez y Vernet 1991).

b) A lo largo del Calcolítico Reciente el clima volvió a condiciones áridas que continuaron durante la primera mitad del II milenio a. C. como ha sido propuesto también por otros autores (Rodríguez y Vernet 1991; Rodríguez 19891990; Capel et al. 1998; Carrión et al. 2007; López-Sáez et al. 2014), incidiendo fuertemente sobre una cobertura vegetal ya objeto de la presión antrópica (Rodríguez et al. 1996; Fuentes et al. 2005).

c) En Los Millares se plantean diferencias de consumo entre grupos sociales e individuos basadas en la distribución diferencial de los restos faunísticos (Navas et al. 2005, 2008) y en un estudio isotópico previo (Waterman et al. 2017). En cualquier caso, para afianzar esta hipótesis, 
deberán estudiarse más muestras de las que hemos podido analizar para este trabajo.

d) La necrópolis estuvo en uso durante todo el periodo de ocupación de Los Millares, es decir, desde el tránsito del IV al III milenio a. C. hasta fines del III (Molina et al. 2004).

\section{MATERIALES Y MÉTODOS}

Las últimas excavaciones arqueológicas en la necrópolis de Los Millares se realizaron en la década de 1950 (Almagro y Arribas, 1963). Desde entonces numerosos estudios se han centrado en su cronología, arquitectura y ajuares funerarios (Blance 1971; Gilman 1976; Chapman 1981, 1990; Castro 1988; Escoriza 1991-1992; Montero 1994; Molina y Cámara 2005; Lozano et al. 2010; Afonso et al. 2011; Peña 2011; Calvín 2014; Maicas 2019).

En esta contribución se presentan 14 nuevas dataciones realizadas sobre una representación de muestras de huesos humanos sometidos a análisis isotópico: 12 proceden de contextos excavados por L. Siret, depositados en el Museo Arqueológico Nacional (MAN) en Madrid, y las otras 2 de restos óseos de las excavaciones de A. Arribas, conservados en el Laboratorio de Antropología de la Universidad de Granada (UGR). Los conjuntos conservados en el Museo Arqueológico de Almería (MAA) se han descartado por el tamaño mínimo de la muestra en la tumba 72 y el retraso en el procesamiento del material de la tumba II/47. Del conjunto analizado, 13 fechas corresponden a tumbas de la Edad del Cobre y una procede de una sepultura del Bronce Final. Las 14 muestras han sido datadas por AMS en el Centro Nacional de Aceleradores (CNA) de Sevilla y calibradas a través del Programa Calib 7.0.2 siguiendo la curva IntCal13 (Reimer et al. 2013) (Tab. 1). Esta primera serie de fechas absolutas disponibles para los inhumados de la necrópolis de Los Millares se suma a la datación del Laboratorio de Köln (KN 72), ya conocida, sobre una muestra de vida larga del tholos 19 (Almagro y Arribas 1963; Molina et al. 2004).

En primer lugar, este amplio conjunto permite discutir propuestas recientes sobre la cronología de los sepulcros del sureste a partir de nuevas dataciones radiocarbónicas (Aranda y Lozano 2014; Lozano y Aranda 2017; Aranda et al. 2017a, 2017b), además, de la contrastación del conjunto con la seriación de las sepulturas de Los Millares basada en sus rasgos formales y en los materiales recuperados (aun teniendo en cuenta el amplio periodo de uso de la mayoría de las tumbas).

Esta combinación de las dataciones con la tipología de tumbas y ajuares contextualiza las 44 muestras con datos positivos en los análisis de isótopos estables (13C y $15 \mathrm{~N})$ del conjunto de 68 seleccionadas. La información isotópica procede de 26 muestras del MAN (Colección Siret), 9 del MAA (Colección Siret 3; excavaciones de M. Almagro y A. Arribas 6) y 9 de la UGR

\begin{tabular}{|c|c|c|c|c|c|c|c|}
\hline $\mathbf{N}^{\mathbf{0}}$ Registro & Tumba & Cod. Laborat. & Fecha BP & $\mathbf{1} \boldsymbol{\sigma}$ cal AC & $\mathbf{2} \boldsymbol{\sigma}$ cal AC & Período & Fase \\
\hline 76-1-Mill-33-13 & Tumba 33 & CNA4434-1-1 & $2680 \pm 30$ & $899-804$ & $896-802$ & BF & BF \\
\hline $76-1-M i l l-51-12$ & Tumba 51 & CNA4435-1-1 & $3690 \pm 30$ & $2134-2032$ & $2195-1977$ & CF & 4 \\
\hline $76-1-M i l l-52-19$ & Tumba 52 & CNA4436-1-1 & $3880 \pm 30$ & $2454-2308$ & $2467-2235$ & CF & 4 \\
\hline $76-1-M i l l-55-340$ & Tumba 55 & CNA4437-1-1 & $4070 \pm 30$ & $2832-2500$ & $2851-2491$ & CT & 3 \\
\hline $76-1-M i l l-56-10$ & Tumba 56 & CNA4439-1-1 & $3970 \pm 30$ & $2564-2465$ & $2574-2350$ & CT & 3 \\
\hline $76-1-M i l l-57-1406$ & Tumba 57.0 & CNA44440-1-1 & $4240 \pm 30$ & $2904-2780$ & $2911-2705$ & CP & 2 \\
\hline $76-1-M i l l-57-1435$ & Tumba 57.1 & CNA4441-1-1 & $4070 \pm 30$ & $2832-2500$ & $2851-2491$ & CP & 2 \\
\hline $76-1-M i l l-57-1449$ & Tumba 57.5 & CNA4444-1-1 & $4100 \pm 30$ & $2840-2579$ & $2862-2503$ & CP & 2 \\
\hline $76-1-M i l l-57-1456$ & Tumba 57.6 & CNA4443-1-1 & $4150 \pm 30$ & $2867-2671$ & $2876-2628$ & CP & 2 \\
\hline $76-1-M i l l-67-32$ & Tumba 67.2 & CNA4445-1-1 & $3980 \pm 30$ & $2564-2469$ & $2577-2457$ & CT & 3 \\
\hline $76-1-M i l l-68-54$ & Tumba 68 & CNA4448-1-1 & $4050 \pm 30$ & $2620-2495$ & $2834-2478$ & CT & 3 \\
\hline $76-1-M i l l-74-57$ & Tumba 74 & CNA4451-1-1 & $4290 \pm 30$ & $2913-2890$ & $3009-2877$ & CA & 1 \\
\hline LM-NEC-XXVI-1-9 & Tumba XXVI/18 & CNA4453-1-1 & $3920 \pm 30$ & $2470-2348$ & $2479-2299$ & CF & 4 \\
\hline LM-NEC-XXVI-5-8 & Tumba XXVI/18 & CNA4457-1-1 & $3540 \pm 30$ & $1931-1781$ & $1953-1768$ & --- & 4 \\
\hline
\end{tabular}

Tab. 1. Relación de las muestras de huesos humanos de la necrópolis de Los Millares con dataciones de C-14. Período: CA Cobre Antiguo; CP Cobre Pleno; CT Cobre Tardío; CF Cobre Final; BA Bronce Antiguo; BF Bronce Final. 


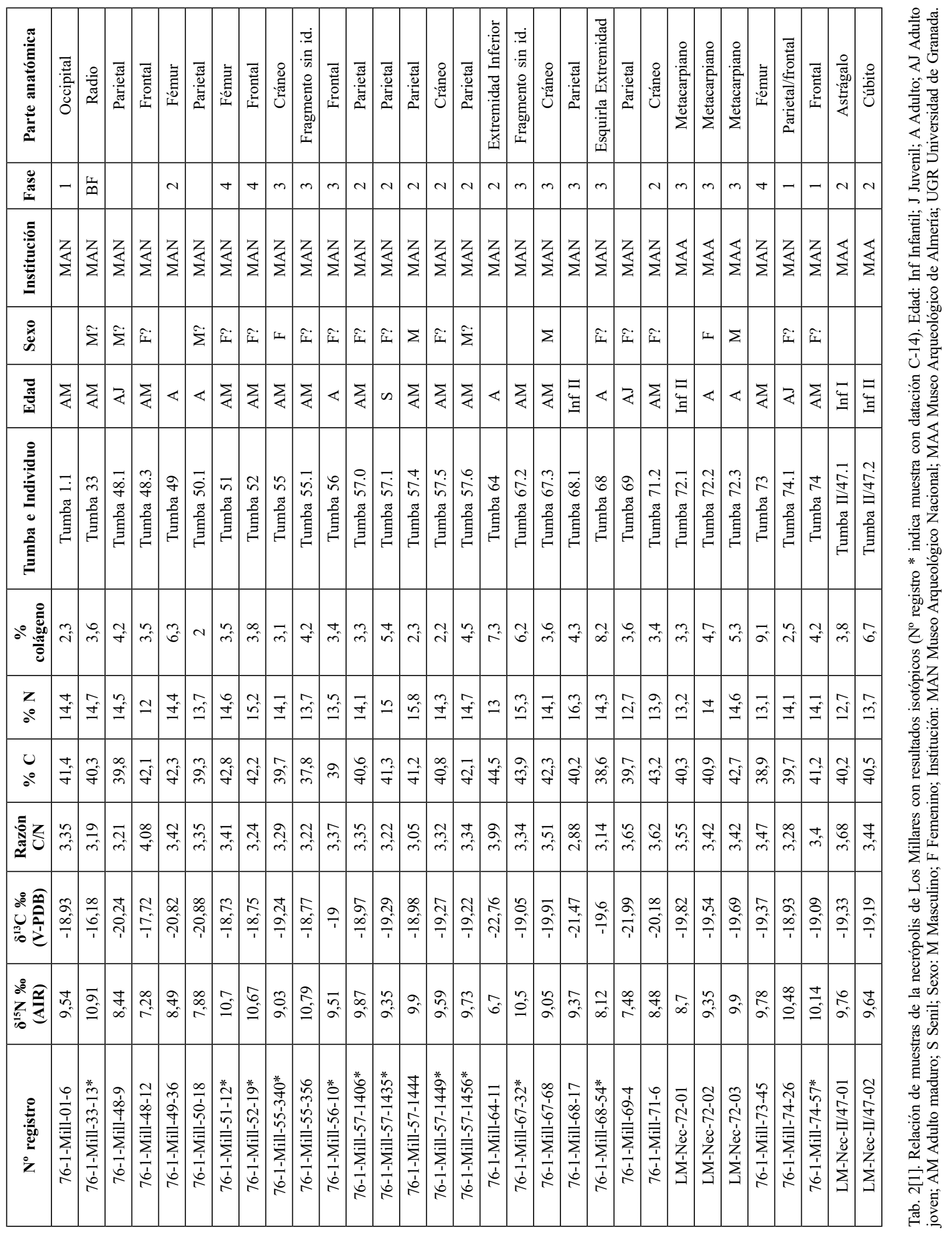




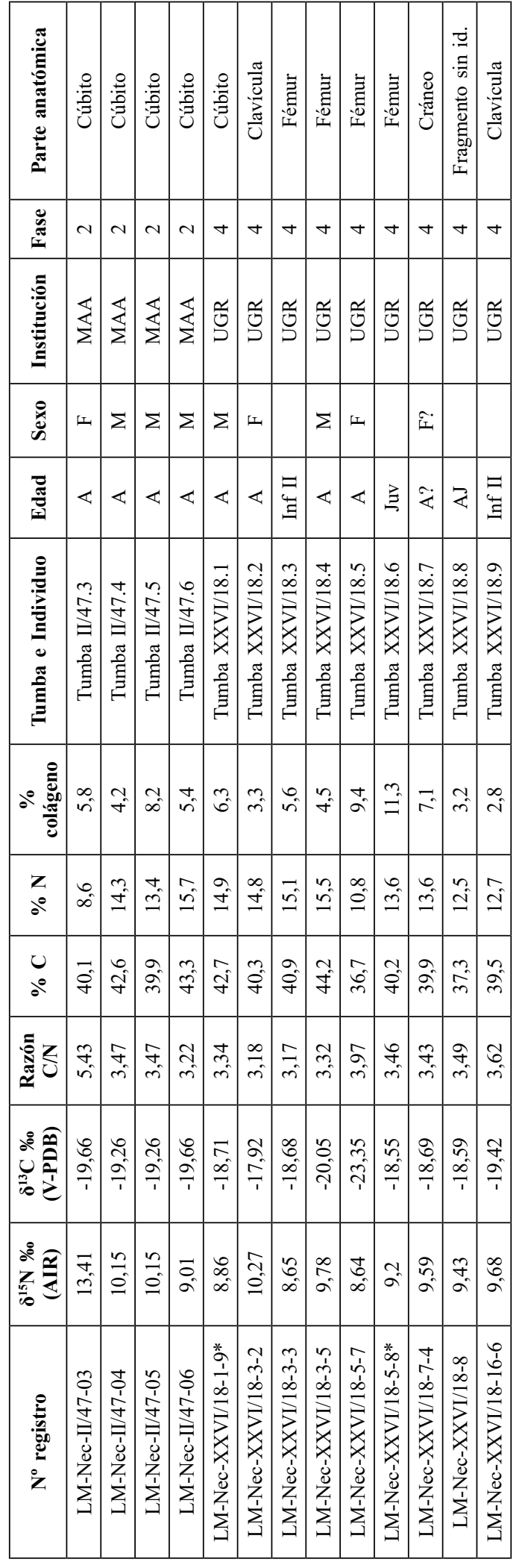

(excavaciones M. Almagro y A. Arribas). En cambio 24 muestras procedentes del MAN carecían de colágeno (Tab. 2).

La selección y clasificación de las muestras obtenidas de la colección de restos humanos de Los Millares se debe a dos de las autoras: V. Peña (MAN) y S. Jiménez-Brobeil (MAA y Laboratorios de la UGR).

La representatividad del conjunto puede apreciarse mejor si nos referimos a las características de la colección más numerosa, la del MAN, y las limitaciones de ciertas muestras para nuestros objetivos. Las muestras de 50 individuos allí conservados suponían un 31,06\% del total disponible, un total limitado por el deseo de tomar muestras que no correspondieran a dientes. Según el estudio preliminar (VP) esta serie antropológica solo conserva huesos de 48 tumbas. El número mínimo de 161 individuos determinados está sesgado hacia los adultos con 103 individuos (entre 19 y más de 55 años), a los que se unen 18 juveniles (13-18 años), 26 infantiles (entre 1 y 12 años), 5 perinatales (desde 7 meses intrauterino a 1 año) y 6 indeterminados.

Es importante la diferencia entre el número de individuos por tumba según los diarios de Flores y según la identificación de los restos óseos del MAN. Flores proporciona un recuento aproximado, ya que los individuos nunca estuvieron en conexión anatómica pero tampoco tenemos la completa seguridad de que el registro actual conservado en los museos, en especial en el MAN, sea todo lo que él encontró. Por ejemplo en la tumba 71 Flores sugiere 20 individuos y en el MAN se conservan 8. A veces, no se conservan los restos antropológicos: Flores sugiere 60 enterramientos en la tumba 5 y 50 en la tumba 7. Finalmente, los huesos conservados pueden ser mínimos como en la tumba 40, donde apenas hay algunos fragmentos de 2 individuos (adulto e infantil), mientras Flores contaba 114 enterramientos.

La determinación del número mínimo de individuos (NMI) conservados en las tumbas colectivas de los Millares ha partido de la identificación de cada resto óseo asignado a ellas, incluida su reconstrucción y su lateralización cuando ello fue posible. Así hemos obtenido para cada tumba el hueso o fragmento más repetido y por tanto el mínimo de individuos documentados.

A su vez los huesos se agruparon para definir un individuo "hipotético" pero compatible atendiendo a criterios de posible edad, sexo, robustez, simetría, continuidad y en algunos casos (siempre como criterio secundario) al aspecto y coloración (deterioro taxonómico). Las esquirlas de hueso y los restos de imposible lateralización se asignaron completando por orden a los individuos mejor representados.

Es importante señalar que la mayoría de los individuos solo están representados por restos de calota craneal, dientes, dedos de manos y pies y, en menor me-

Trab. Prehist., 77, N. ${ }^{\circ}$ 1, enero-junio 2020, pp. 67-86, ISSN: 0082-5638

https://doi.org/10.3989/tp.2020.12247 
dida, pequeños fragmentos de huesos largos. Para tomar muestras seguras de individuos diferentes se seleccionaron huesos perfectamente identificados y lateralizados. Normalmente se recogieron muestras del mismo metacarpo o metatarso (mismo hueso, mismo lado) repetidos y por tanto incompatibles entre sí o procedentes de individuos reales diferenciados. En algún caso, si se conserva más completo un cráneo, se eligió la muestra también del mismo hueso. Eso sucedió en la tumba 57 donde 4 muestras proceden de parietales: tres de adultos diferentes y la cuarta del cráneo de un individuo senil con todos los huesos unidos, sin marcas de sutura (“cráneo incompleto", signatura 76-IMill/57-1449). Cuando era imposible seleccionar el mismo hueso, se escogió otro fragmento de individuo infantil o juvenil, completamente incompatible con el resto de muestras. P. ej., en la tumba 68 una muestra procedía de un hueso largo adulto (76-I-Mill/68-54) y otra de un fragmento de parietal de un cráneo infantil II, probablemente entre 7-8 años (76-I-Mill/68-17), es decir, pertenecían claramente a individuos diferentes.

Como se ha dicho, 24 de las muestras del MAN carecían de suficiente colágeno. Ello ha puesto en duda su grado de preservación para el análisis de isótopos estables y/o para su datación por C14. La falta de colágeno se debía a que todos los huesos humanos se hallaban muy quemados, sin deformaciones o fragmentaciones transversales, indicando que el fuego actuó cuando el cuerpo estaba ya esqueletizado y el hueso seco. Estos fuegos serían el resultado de los continuos procesos de limpieza in situ de los paquetes óseos acumulados para liberar espacio, además de otras actividades rituales realizadas en el interior de las sepulturas colectivas, no quedando casi constancia de huesos con abundante tejido esponjoso como vértebras o costillas que serían consumidos por el fuego (Maicas 2005; Peña 2011).

Además los resultados de 4 de las 44 muestras con información no son fiables por tener ratios entre los isótopos de carbono y nitrógeno superiores a 3,7. En la discusión de los resultados se valorará el conjunto con y sin los datos de estas 4 muestras.

Los análisis isotópicos han seguido el procedimiento estándar del Laboratorio de Biogeoquímica de Isótopos Estables del Instituto Andaluz de Ciencias de la Tierra (CSIC-UGR), siguiendo las recomendaciones de Bocherens et al. (1997). Primero el colágeno se extrajo de las muestras óseas por un procedimiento que aseguró la eliminación de otros componentes orgánicos e inorgánicos. Para ello las muestras se limpiaron raspándolas y después se redujeron a pequeños fragmentos. Tras ello, se desmineralizaron (fosfatos y carbonatos) $800 \mathrm{mg}$ de material óseo en $\mathrm{HCl} 1 \mathrm{M}$ durante $20 \mathrm{mi}$ nutos a temperatura ambiente. En un primer filtrado se suprimieron ácidos fúlvicos. Posteriormente el residuo se llevó a un pH básico con una solución de $0,125 \mathrm{M}$ $\mathrm{NaOH}$, de modo que al pasar por un segundo filtro (MF-Millipore de $5 \mu \mathrm{m}$ ) quedó retenido el colágeno (y restos de silicatos, esencialmente algún resto de arcilla), resultando eliminados posibles restos de ácidos húmicos y otros ácidos solubles en $\mathrm{pH}$ básico. En un tercer paso, el residuo presente en el filtro, sobre todo colágeno (y algún residuo de arcilla, como se ha dicho), se sometió a un tratamiento de $0,01 \mathrm{M} \mathrm{ClH}$ en tubos de Pyrex cerrados a $100{ }^{\circ} \mathrm{C}$ durante 17 horas, de modo que se solubilizó el colágeno. Se centrifugó (6300 rpm durante 10 minutos) para separar la arcilla y otros silicatos que permanecen en el fondo y el sobrenadante (colágeno disuelto) se llevó a otro recipiente que se congeló a $-20{ }^{\circ} \mathrm{C}$ y se liofilizó de modo que se concentrase el colágeno. El colágeno ya purificado se guardó a $-20{ }^{\circ} \mathrm{C}$ hasta su análisis.

Para los análisis isotópicos de carbono y nitrógeno se pesaron alrededor de $0,7 \mathrm{mg}$ de colágeno dentro de una cápsula de estaño por duplicado, y se utilizó un analizador elemental (Carlo Erba Model NA1500 NC) para la combustión de la muestra. El $\mathrm{N}_{2}$ y el $\mathrm{CO}_{2}$ fueron arrastrados por helio hacia una columna cromatográfica y ya separados entraron en un espectrómetro de masas (Delta Plus XP), en el que se analizan las razones isotópicas de carbono y nitrógeno. Cada 5 muestras se intercalaron patrones internos, que previamente habían sido calibrados con patrones internacionales (IAEA-N-1, IAEA-NO-3, IAEA-N-2, USGS34, USGS35, USGS25, USGS61, USGS62). Los patrones internos seleccionados EEZ-26 $\left(\delta^{13} \mathrm{C}=-30,09 ; \delta^{15} \mathrm{~N}=\right.$ $-2,13 \%$ ) y EEZ-23 $\left(\delta^{13} \mathrm{C}=-13,27 ; \delta^{15} \mathrm{~N}=+16,10 \%\right.$ ) cubren todo el rango posible de valores isotópicos de las muestras. Los resultados no se obtienen en valores absolutos, sino en función de las diferencias con los valores de referencia de composición internacional $(\delta)$ aceptados. El error analítico para las determinaciones $\delta^{15} \mathrm{~N}$ y $\delta^{13} \mathrm{C}$ es $<0,1 \%$.

Los estudios de isótopos estables sobre restos humanos de la Prehistoria Reciente procedentes de yacimientos de la fachada mediterránea y del sur de la península ibérica son cada vez más frecuentes (Van Strydonck et al. 2005; García-Guixé et al. 2006; Salazar-García 2009, 2011; Salazar-García et al. 2014a, 2014b, 2016, 2018; Salazar-García y Pinto 2017; Fuller et al. 2010; Díaz-Zorita 2013; Díaz-Zorita et al. 2011, 2014, 2017, 2018, 2019; McClure et al. 2011; Nehlich et al. 2012; Fernández-López et al. 2013; FontanalsColl et al. 2014, 2017; Gibaja et al. 2015; Molina et al. 2016, 2019; Esparza et al. 2017; Waterman et al. 2017; Beck et al. 2018; Villalba-Mouco et al. 2018), aunque el conjunto de muestras en cada estudio y para cada fase cronológica suele ser reducido.

Especialmente relevante para nuestro trabajo es el estudio de Waterman et al. (2017) de 12 muestras pro- 
cedentes de 4 tumbas $(55,57,74$ y 63$)$ de la propia necrópolis de Los Millares, tres de las cuales $(55,57$ y 74) se fechan en este trabajo. Ello les proporciona un marco cronológico más preciso, aunque no ha habido suficiente colágeno para datar la muestra de la tumba 63. A diferencia del trabajo precedente que usa el carbonato asociado al apatito, nosotros nos hemos centrado en el colágeno del hueso ya que no existe una buena correlación entre $\delta^{13} \mathrm{C}$ del carbonato asociado al apatito presente en el esmalte del diente y la dieta. Esto se debe a que el bicarbonato disuelto en la sangre tiene dos fuentes: la dieta ingerida y el bicarbonato presente en el agua que beben los individuos. Por consiguiente, cuando se correlacionan ambos valores se suele obtener una gran variabilidad en datos procedentes del carbonato presente en el esmalte frente a una mayor consistencia en los datos de colágeno del hueso. A este factor, se le sumarían anomalías relacionadas con procesos diagenéticos: los iones carbonato y bicarbonato presentes en el suelo pueden recristalizarse en el esmalte, afectando a la señal isotópica primaria, lo que no ocurre con el colágeno.

Las principales diferencias entre el trabajo de Waterman y otros y el aquí realizado se resumen en el material analizado: la apatita de los dientes en el suyo frente al colágeno extraído de huesos en nuestro caso. Junto a ello, cabe valorar el tamaño mayor de la muestra analizada en el presente trabajo, la adición de materiales procedentes de las excavaciones de los años 1950 depositados en el Museo Arqueológico de Almería y en la Universidad de Granada y, sobre todo, su contextualización cronológica a través de las tipologías sepulcrales y fundamentalmente de las dataciones absolutas obtenidas de los restos óseos de los individuos inhumados. Ello representa la única forma de apreciar variaciones ambientales y de dieta, e ir más allá de la constatación de una dieta principalmente basada en recursos terrestres como se ha demostrado (Waterman et al. 2017), planteándose, aun así, un consumo de peces que debió ser esporádico, a juzgar por los elementos de ictiofauna detectados en los depósitos del yacimiento (Peters y Driesch 1990).

\section{RESULTADOS Y DISCUSIÓN}

\subsection{La cronología de la necrópolis}

La probabilidad conjunta (sum of probabilities) de las 13 fechas radiocarbónicas disponibles para los restos humanos de la necrópolis de Los Millares, excluyendo la datación de la sepultura del Bronce Final, ofrece un intervalo máximo a $1 \sigma$ de 2918 y $2346 \mathrm{cal}$ $\mathrm{AC}$ y a $2 \sigma$ entre 2924 y 1778 cal AC, usando el programa Calib 7.0.4. Frente a lo que sucede en el rango
$1 \sigma$, en el rango $2 \sigma$ la situación es más compleja como resultado de dos dataciones que alcanzan fechas que entran en el II milenio a. C., principalmente CNA4457 $(3540 \pm 30 \mathrm{BP})$. Corresponde a la tumba XXVI, excavada pero no publicada por A. Arribas y M. Almagro, donde se ha obtenido otra fecha del mismo contexto con una datación más acorde a los esperado. En cualquier caso, al 93,67 \% de probabilidad dentro del rango $2 \sigma$, se puede considerar que la necrópolis se usa hasta el final del III milenio (media 2026) cal AC.

De la campaña de 1956, Almagro y Arribas tomaron una muestra de vida larga procedente de la tumba 19, KN-72 (Schwabedissen y Freundlich 1966: 241), que en su media marca un momento del Calcolítico Antiguo $(4380 \pm 120 \mathrm{BP})$. Entre las dataciones aquí presentadas y realizadas sobre huesos humanos, solo el rango $2 \sigma$ de la datación CNA4451 se aproxima a esa fecha.

Estos intervalos coinciden bastante bien con lo propuesto para el conjunto del yacimiento en función de las fechas disponibles para el poblado y los fortines (Molina et al. 2004) y que sitúan la ocupación de Los Millares entre 2887 y $2207 \mathrm{cal} \mathrm{AC}$ en el rango $1 \sigma$, que se amplía a 3320 y 2144 en el rango $2 \sigma$, situando la ocupación entre 3113 y 2193 cal AC con un intervalo a un $95,81 \%$ de probabilidad.

Además la fecha CNA4435 (3690 $\pm 30 \mathrm{BP})$ sobre huesos humanos, correspondiente a la tumba 51, se sitúa muy a fines del III milenio cal AC. Puede ser contemporánea con la fase inicial de la Cultura de El Argar en las depresiones de Vera y especialmente en la comarca de Lorca, pero es anterior a la introducción de esta cultura de la Edad del Bronce en el valle del Andarax. De esta tumba apenas hay datos en la publicación del matrimonio Leisner, señalándose solo la atribución a la fase III de Siret y su tipología (rundgräber sin corredor), Su único ajuar, una lámina de sílex, no concuerda con una atribución al Bronce Antiguo (Leisner y Leisner 1943: 53-54).

La obtención de fechas del II milenio a. C. en la necrópolis podría apoyar las propuestas sobre la reutilización de los sepulcros, que se constata en otras áreas del sureste para momentos iniciales y medios de la Edad del Bronce (Aranda y Lozano 2014; Lozano y Aranda 2017), en consonancia con otras reutilizaciones megalíticas en distintas áreas europeas (DíazGuardamino et al. 2015). Sin embargo, en la necrópolis de Los Millares solo la tumba 28, por su ajuar funerario, podría fecharse en la Edad del Bronce. Es un pequeño megalito ortostático de cámara trapezoidal, al que Siret asoció con un puñal con remaches (Leisner y Leisner 1943: 53, lám. 24/7). Junto a otros dos megalitos, se situaría en una zona periférica de la necrópolis denominada por Siret "Pisada de la Virgen",

Trab. Prehist., 77, N. ${ }^{\circ}$ 1, enero-junio 2020, pp. 67-86, ISSN: 0082-5638

https://doi.org/10.3989/tp.2020.12247 
cuya relación con el asentamiento de Los Millares no está contrastada.

Otro aspecto a considerar respecto a la cronología es la posibilidad de aproximarnos al periodo de uso de algunos sepulcros concretos, gracias a la obtención de varias fechas en algunos de ellos.

La tumba 57 es el primer caso. Presentaba cámara circular con corredor de dos tramos y al menos 30 cadáveres, según G. y V. Leisner (1943: 32), que serían 40 con la revisión antropológica. Ofrece el ajuar más relevante de las tumbas muestreadas: 48 puntas de flecha y numerosas hojas en piedra tallada, cuentas de collar, ídolos falange, tolva y antropomorfos, hacha de metal y cerámica simbólica (Leisner y Leisner 1943: 32, lám. 14/2). G. y V. Leisner (1943: 566) la sitúan en su fase Ib. La probabilidad conjunta de las 4 fechas obtenidas sitúan su uso entre 2902 y $2575 \mathrm{cal} \mathrm{AC}$ en el intervalo a $1 \sigma$ y entre 2904 y 2503 a $2 \sigma$ (con un fin a 2571 en el $98,2 \%$ de ese rango), dentro del Calcolítico Pleno.

La tumba XXVI, la segunda para la que contamos con dos dataciones, fue correlacionada con dudas con la tumba 18 de las excavaciones de Siret (Almagro y Arribas 1963: 51-54), aunque tal identificación no fue recogida por R. Chapman (1981: 79). De ser cierta la correlación, se trataría de un sepulcro de cámara circular con corredor de 3 tramos y vestíbulo que contenía más de 20 cadáveres. Su ajuar con 8 puntas de flecha en piedra tallada, láminas de sílex, cuentas de collar y cerámica campaniforme de tipo marítimo fue incluido en la fase Id (Leisner y Leisner 1943: 39, 567, lám. 18/1). Si bien se muestrearon varios individuos, solo dos han podido ser datados. Como una de ellas, la CNA4457, resulta sorprendentemente tardía, la probabilidad conjunta de ambas ha ofrecido un amplio periodo de uso para el sepulcro: entre 2471 y 1782 a $1 \sigma$ y entre 2479 y 1770 a $2 \sigma$. También se debe considerar que tres de las nueve muestras que ofrecieron colágeno para su análisis isotópico han tenido problemas en la razón $\mathrm{C} / \mathrm{N}$ y han sido excluidas del estudio. Podemos desconfiar especialmente de la datación CNA4457. En cualquier caso, frente a las dataciones $\mathrm{y}$ ajuar funerario del sepulcro anterior, las fechas y materiales de este segundo sepulcro apoyan su uso en la segunda mitad del III milenio a. C., pero siempre en un contexto calcolítico.

Además hemos asignado la tumba 55 al Cobre Tardío (datación CNA4437-1-1), aunque otra fecha de C14 idéntica (CNA4441-1-1) afecta a la tumba 57 que hemos atribuido al Cobre Pleno, basándonos en el ajuar funerario y en otras dos dataciones realizadas en la misma tumba que solo pueden atribuirse a este período. Nuestra asignación de la tumba 55 se basa en su adscripción por G. y V. Leisner (1943: 567) a la fase Id por su tipología constructiva y su ajuar poco dis- criminante, así como por el arco de la calibración de su datación, transicional entre ambos periodos.

El episodio final para el uso funerario del área viene marcado por la fecha CNA4434 $(2680 \pm 30 \mathrm{BP})$, correspondiente a un enterramiento del Bronce Final, la tumba 33, cuya tipología constructiva, materiales y cronología carece de relación directa con la necrópolis calcolítica de Los Millares (Siret 1893: 75, figs. 300301; Leisner y Leisner 1943: 53, lám. 24/8; Lorrio 2008: 152-154, figs. 86-87).

\subsection{La seriación de las muestras del estudio isotópico}

Estos resultados de $\mathrm{C} 14$ y la tipología y contenido de los sepulcros nos permiten una ordenación cronológica de las tumbas muestreadas que aún debe ser tomada con ciertas precauciones por la escasez de las fechas disponibles.

Según las dataciones de C14 obtenidas en el poblado y la necrópolis, se podría plantear que la fundación de Los Millares se remonta aproximadamente al 3100 cal AC. Al Cobre Antiguo (transición entre el IV y el III milenio a. C., 3100-2900 cal AC) hemos atribuido las muestras de las tumbas 1 y 74 . También se ha conseguido una datación antigua para la tumba 57 que se mantuvo en uso durante el Cobre Pleno. G. y V. Leisner (1943: 566) situaron la tumba 1 en su fase Ia, dejando sin adscribir la tumba 74, para la que disponemos de una datación radiocarbónica congruente con la propuesta. Por último, los Leisner (1943: 566) asignaron a la fase Ib la tumba 57, cuyo uso debió ser prolongado. Las tumbas 1 y 74 son sepulcros de corredor corto, que ofrecen en torno a la decena de cadáveres y una cantidad similar de puntas de flecha, algunas de pedúnculo y aletas o romboidales, cerámicas con decoración simbólica, vasos cilíndricos y cuencos de borde entrante. La 74 tiene ídolos tolva y falange y un peine de hueso-marfil (Leisner y Leisner 1943: 24, lám. 10/2) y la tumba 1 un ídolo cilíndrico (Leisner y Leisner 1943: lám. 8/1). La dudosa correlación de la tumba 74 con la XIII de las excavaciones de A. Arribas (Almagro y Arribas 1963: 54-55; Chapman 1981: 79) no nos parece aceptable ni por los materiales ni por la datación.

Al Cobre Pleno (primer tercio del III milenio a. C. 2900-2600 cal AC) hemos adscrito las tumbas II (47 de Siret) y 57, atribuidas respectivamente a las fases Ic y Ib de G. y V. Leisner (1943: 566). Las fechas disponibles para la tumba 57 demuestran la continuidad hasta la fase siguiente, característica de la mayoría de estos sepulcros colectivos. Son tumbas de corredor medio dividido en tramos y con nichos. Ambas tienen cerámica simbólica y vasos con formas cilín- 
dricas y ovoides, ídolos tolva y falange y metal (en las dos). El número de inhumados es variable. En la tumba 57 superan la treintena, llegando las puntas de flecha a 48, también con casos romboidales y de pedúnculo y aletas. La II-47 presenta incluso un peine (Leisner y Leisner 1943: 43, lám. 19/2).

Al Cobre Tardío (siglos centrales del III milenio a. C., 2600-2400 cal AC) se atribuyen las muestras de las tumbas $48,49,50,55,56,67,68,69,71,72$ y 73 . A la fase Id de G. y V. Leisner se adscribieron las tumbas $49,55,67,71$ y 73 y a la fase II las tumbas 48, 50, 56, 68 y 72 (Leisner y Leisner 1943: 567; Chapman 1981: 82). Las tumbas 68 y 72 se identifican respectivamente con las sepulturas XXX y XVII, mientras se duda entre la 69 y la 71 para una correlación con la XIV (Almagro y Arribas 1963: 54-55). Disponemos de dataciones para las tumbas 55, 56, 67 y 68 cuyos corredores son más largos y con segmentaciones doble, triple o cuádruple. El ajuar recuperado es escaso: pocas hojas y puntas de flecha de sílex y cuentas de diverso tipo, acompañando a pocos cadáveres. Solo se refiere un número alto de inhumados en las tumbas 67,69 y $71(6,5$ y 8 cadáveres respectivamente según la revisión antropológica). En la tumba 71 se ha localizado un ídolo-falange (Leisner y Leisner 1943: 45, lám. 21/1).

Al Cobre Final (tercer cuarto del III milenio a. C., 2400-2100 cal AC) se adscriben las tumbas 51, 52 y XXVI, esta última correlacionada con la 18 con muchas dudas (Almagro y Arribas 1963: 51, 54). La 51 no fue adscrita a ninguna fase por los Leisner, que consideraron la 52 de la fase II (Leisner y Leisner 1943: 567). Tenemos dataciones para las tres tumbas y aparece un vaso campaniforme marítimo en la XXVI (Leisner y Leisner 1943: 39, lám. 18/1), si aceptamos la correlación. La complejidad de las tumbas, en general, es mayor con recintos externos para betilos, nichos y vestíbulos ya desde el Cobre Tardío. Como en casi todas las tumbas donde se nos ha permitido obtener muestras, el ajuar es escaso. Destaca la presencia de cerámica campaniforme. La supuesta presencia de 25 enterramientos en la sepultura 18-XXVI es difícil de valorar al no conservarse apenas huesos en el MAN.

Finalmente, como se ha dicho, también se dató la tumba 33 (Siret 1893: 75, figs. 300-301; Leisner y Leisner 1943: 53, lám. 24/8), perteneciente a la necrópolis del Bronce Final, superpuesta espacialmente a la necrópolis del asentamiento calcolítico. Sin embargo sus características tipológicas, con pequeñas sepulturas en cistas megalíticas de planta cuadrada, rectangular o circular y sin corredor, son muy distintas a las de las tumbas de la Edad del Cobre.

Para minimizar los problemas de continuidad y los errores en la atribución cronológica de los sepulcros, los valores isotópicos serán discutidos también teniendo en cuenta las diferencias cronológicas entre los individuos datados de la misma tumba. Solo se han considerado las muestras directamente datadas para ver si se confirman o no las tendencias apreciadas en el estudio global, que incluye también las muestras no datadas por C14, según la atribución cronológica planteada en los párrafos anteriores.

\subsection{Resultados de los análisis isotópicos}

Los valores $\delta^{15} \mathrm{~N}$ están comprendidos entre 6,70 y $13,41 \%$ (AIR) mientras que los valores $\delta^{13} \mathrm{C}$ oscilan entre $-23,35$ y $-16,18 \%$ (V-PDB). Si atendemos a los valores de todas las muestras que ofrecieron datos isotópicos (Tab. 2), la media en los valores $\delta^{15} \mathrm{~N}$ es de $+9,45 \%$ (AIR) con una desviación típica de 1,12, mientras la media en $\delta^{13} \mathrm{C}$ es de $-19,47$ con una desviación típica de 1,23 .

Atendiendo a la relación entre los isótopos de carbono y nitrógeno, existen 4 casos anómalos (proporción superior a 3,7). Al suprimirlos la distribución de los valores $\delta^{15} \mathrm{~N}$ se ve más afectada aumentando sensiblemente su concentración alrededor de la media (Tab. 3). En ambos casos existe un alto grado de estandarización que apunta hacia una importante fiabilidad de los datos.

Según el estudio de la normalidad en los datos, las distribuciones de ambos isótopos no muestran diferencias estadísticamente significativas con la distribución normal $\left(Z=0,583, \alpha=0,886\right.$ para $\delta^{15} \mathrm{~N}$ y $Z=0,974$, $\alpha=0,299$ para $\delta^{13} \mathrm{C}$ ), hipótesis necesaria para la aplicación de técnicas de inferencia estadística.

Destacamos que encontramos los valores más altos de $\delta^{15} \mathrm{~N}$ en las tumbas $33,55,51,52,67$ y 74 , en algunos individuos de la tumba II y en uno de los individuos de la tumba XXVI. Los factores cronológicos pueden explicar la variabilidad, como después discutiremos, pero puede resultar significativo que, al menos en las primeras fases, los individuos inhumados en las tumbas que han conservado más ajuar mostraran valores que podrían sugerir el consumo de una proporción ligeramente mayor de proteínas animales, aunque estos valores también podrían estar afectados por variables ambientales.

\begin{tabular}{|c|c|c|c|c|c|}
\hline & Mínimo & Máximo & Media & $\begin{array}{c}\text { Desviación } \\
\text { Típica }\end{array}$ & $\begin{array}{c}\text { Coeficiente } \\
\text { de } \\
\text { Variación }\end{array}$ \\
\hline $\boldsymbol{\delta}^{\mathbf{1 5}} \mathbf{N}$ & 7,48 & 10,91 & 9,49 & 0,81 & 0,0851 \\
\hline $\boldsymbol{\delta}^{\mathbf{1 3}} \mathbf{C}$ & $-21,99$ & $-16,18$ & $-19,33$ & 0,95 & 0,0493 \\
\hline
\end{tabular}

Tab. 3. Estadísticos descriptivos de los indicadores $\delta^{15} \mathrm{~N}$ y $\delta^{13} \mathrm{C}$ de las muestras de la necrópolis de Los Millares. 
Según los resultados obtenidos al considerar la división entre sexos (Fig. 3) no hay diferencias estadísticamente significativas en media entre individuos masculinos y femeninos tal y como muestra el test tStudent $\left(\mathrm{t}=0,583, \alpha=0,565\right.$ para $\delta^{15} \mathrm{~N}$ y $\mathrm{t}=0,861$, $\alpha=0,398$ para $\delta^{13} \mathrm{C}$ ). Ello parece en consonancia con los pocos datos disponibles para la fase calcolítica del yacimiento de Eras del Alcazar de Úbeda (Molina et al. 2019) y que se ha propuesto para Marroquíes (Jaén) (Beck et al. 2018). Sin embargo, en la representación gráfica se observa una cierta tendencia a valores superiores a la media en $\delta^{13} \mathrm{C}$ en los individuos femeninos. El individuo aislado (localizado en la parte superior-derecha) corresponde a la sepultura del Bronce Final.

Con respecto a la edad (Tab. 4), la mayoría de los individuos son adultos (casi un $73 \%$ ), incluyendo un único senil. Un factor adicional reseñable es que no se aprecian valores en $\delta^{15} \mathrm{~N}$ sustancialmente altos en los infantiles, ni siquiera en aquellos que deberían estar todavía en la lactancia, aunque sí se observa un descenso en los adultos jóvenes. Esto difiere de lo que suele observarse en muestras del Bronce Pleno del sureste de la península ibérica y de La Mancha (Molina et al. 2016, 2019) y de lo que se ha referido para los yacimientos calcolíticos de Marroquíes (Jaén) (Beck et al. 2018) y la Arruzafa (Córdoba) (Martínez et al. 2020), o incluso para yacimientos portugueses (Waterman et al. 2014).

La aplicación del test ANOVA (Venables y Ripley 2002; Sokal y Rohlf 2012) no muestra diferencias significativas en los valores isotópicos respecto a los intervalos de edad $(\mathrm{F}=0,984, \mathrm{gl}=3, \alpha=0,417$ para $\delta^{15} \mathrm{~N}$ y $\mathrm{F}=1,178, \mathrm{gl}=3, \alpha=0,339$ para $\left.\delta^{13} \mathrm{C}\right) . \mathrm{La}$ evolución de la media del porcentaje en $\delta^{15} \mathrm{~N}$ disminuye en los adultos-jóvenes y aumenta drásticamente en los adultos. La evolución de la media del porcentaje en $\delta^{13} \mathrm{C}$ indica unas pequeñas cantidades en las edades tempranas (infantiles y adultos-jóvenes) y un aumento significativo en los adultos. Sin embargo, hay que tener en cuenta que la muestra es muy reducida, especialmente en el caso de los seniles.

El comentario de los resultados por periodos cronológicos exige recordar que algunas tumbas carecen de dataciones y que, como sepulturas colectivas que son, a menudo permanecieron en uso durante mucho tiempo. Además las tumbas 48, 50 y 69 tienen ajuares poco característicos. Ello dificulta su adscripción segura a un período concreto, aunque se han asignado a fases antiguas de la secuencia. Para minimizar estos problemas, se considerarán de dos formas los valores isotópicos de las muestras atribuidas a cada uno de los periodos en que se ha dividido la secuencia de Los Millares (Cobre Antiguo, Cobre Pleno, Cobre Tardío y Cobre Final).
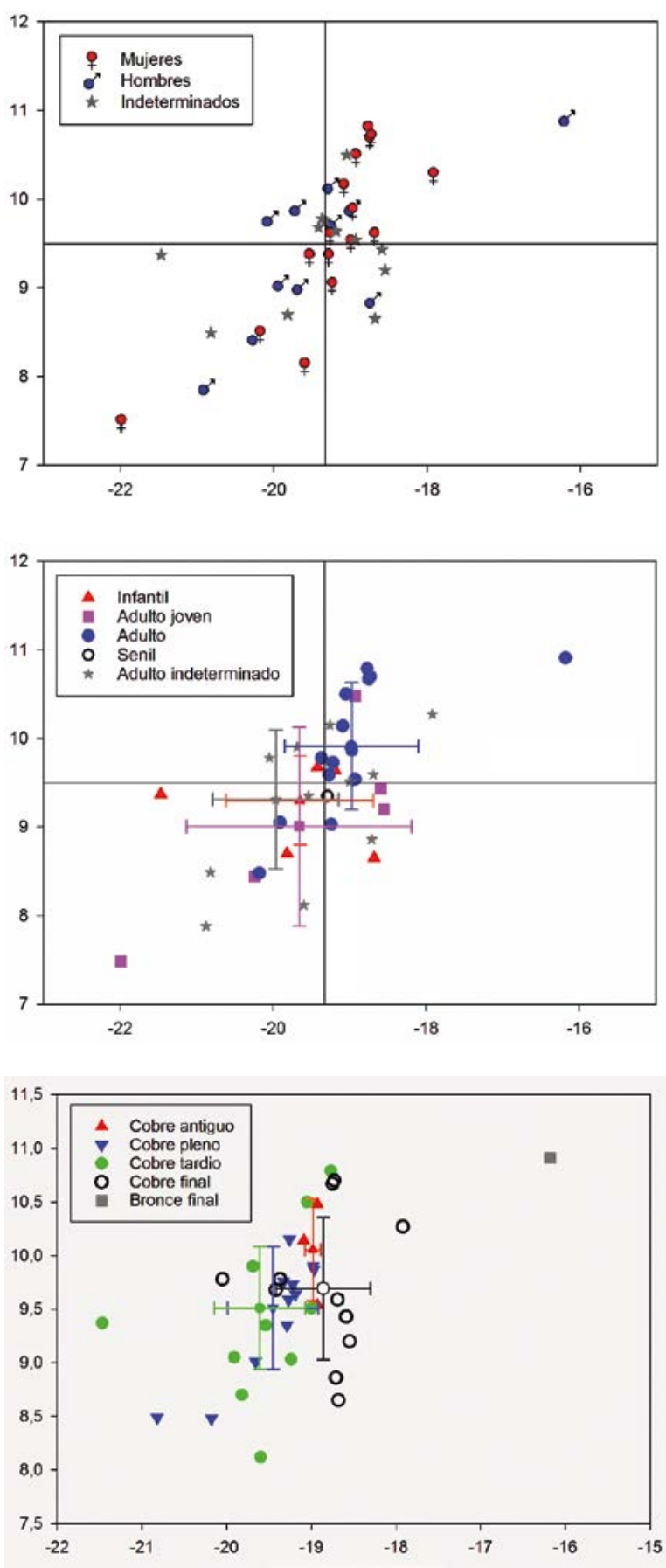

Fig. 3. Diagramas de dispersión de $\delta 15 \mathrm{~N}$ frente a $\delta 13 \mathrm{C}$ de las tumbas estudiadas de la necrópolis de Los Millares. Arriba, relación entre individuos masculinos y femeninos; en el centro, relación entre las diferentes categorías de edad; y abajo, distribución respecto a las fases cronológicas. Las cruces representan las desviaciones estándar de las variables en las distintas categorías con su media en el punto dónde se unen ambas líneas (en color en la versión electrónica). 


\begin{tabular}{|l|c|c|}
\hline \multicolumn{1}{|c|}{ Categoría (Edad) } & Frecuencia & Porcentaje \\
\hline Infantil (0-12) & 6 & 15 \\
\hline Juvenil y A. joven (13-29) & 5 & 12,5 \\
\hline Adulto maduro (30-55) & 15 & 37,5 \\
\hline Senil (>56) & 1 & 2,5 \\
\hline Adulto indeterminado & 13 & 32,5 \\
\hline
\end{tabular}

Tab. 4. Individuos por intervalos de edad de las muestras de la necrópolis de Los Millares.

En primer lugar, se expondrán las medias de todas las muestras atribuidas a cada periodo y, a continuación, se señalarán las medias del conjunto de muestras datadas por radiocarbono correspondientes a cada periodo. Del análisis con pruebas de significación de las diferencias entre los periodos se excluyen las tumbas sin datación y las muestras que presentan una proporción carbono/nitrógeno anómala. Es decir se trabaja con 37 individuos cuya distribución de frecuencias aparece en la tabla 5.

\begin{tabular}{|l|c|c|}
\hline \multicolumn{1}{|c|}{ Fase } & Frecuencia & Porcentaje \\
\hline Cobre Antiguo & 3 & 8,1 \\
\hline Cobre Pleno & 12 & 32,4 \\
\hline Cobre Tardío & 10 & 27,0 \\
\hline Cobre Final & 11 & 27,7 \\
\hline Bronce Final & 1 & 2,7 \\
\hline
\end{tabular}

Tab. 5. Distribución por periodos de los individuos de las muestras de la necrópolis de Los Millares.

La muestra de 37 individuos continúa ajustándose a una distribución normal (contraste de KolmogorovSmirnov usando el método de Monte Carlo: $\mathrm{Z}=0,577$, $\alpha=0,863$ para $\delta^{15} \mathrm{~N}$ y $Z=1,057, \alpha=0,194$ para $\delta^{13} \mathrm{C}$ ). La correlación total entre las muestras $(r=0,755)$ revela una tendencia lineal determinada por el Cobre Pleno y el Cobre Tardío. En cambio en las otras fases no se relacionan los valores de ambos isótopos, ya sea por la escasez de datos (Cobre Antiguo y Bronce Final) o por el bajo valor de la correlación (Cobre Final) (Fernández 2015).

Para la aplicación de la ANOVA se descarta el individuo del Bronce Final por no ser representativa una muestra de tamaño 1. La aplicación del contraste ANOVA indica que no existen diferencias significativas en los valores isotópicos de $\delta^{15} \mathrm{~N}$ respecto al periodo cronológico $(\mathrm{F}=0,811, \mathrm{gl}=3, \alpha=0,497)$, mientras que para $\delta^{13} \mathrm{C}$ se rechaza la hipótesis de igualdad de medias en las diferentes fases $(\mathrm{F}=3,386, \mathrm{gl}=3, \alpha=0,030)$. La aplicación del contraste post hoc de Tukey muestra únicamen-

\begin{tabular}{|c|c|c|c|c|}
\hline & CA & CP & CT & CF \\
\hline CA & 1 & 0,623 & 0,400 & 0,989 \\
\hline CP & & 1 & 0,928 & 0,104 \\
\hline CT & & & 1 & $\mathbf{0 , 0 3 5}$ \\
\hline CF & & & & 1 \\
\hline
\end{tabular}

Tab. 6. Valores de significación del contraste post hoc de Tukey para la muestra de 36 individuos calcolíticos. El asterisco indica que entre $\mathrm{CT}$ y $\mathrm{CF}$ aparecen diferencias estadísticamente significativas. Período: CA Cobre Antiguo; CP Cobre Pleno; CT Cobre Tardío; CF Cobre Final.

te diferencias significativas (nivel de significación $\alpha<0,05$ ) entre las fases Cobre Tardío y Cobre Final (Tab. 6).

La evolución de la media del porcentaje en $\delta^{15} \mathrm{~N}$ muestra una disminución hasta Cobre Tardío con una subida importante hasta el Cobre Final, aunque estas diferencias no son estadísticamente significativas (Fig. 4). Atendiendo a la evolución de la media del porcentaje en $\delta^{13} \mathrm{C}$, el patrón temporal es similar pero la subida en media entre Cobre Tardío y Final es aún más pronunciada y, en este caso, el contraste de Tukey muestra diferencias estadísticamente significativas entre estas dos fases, como hemos dicho. Valores más negativos en $\delta^{13} \mathrm{C}$ pueden asociarse a una mayor humedad y menor WUE (Water-use efficiency) en las plantas.

Ello sugiere una tendencia hacia el aumento en la aridez en línea con lo sugerido a partir de otros análisis para el mismo período en el cuadrante sureste de la península ibérica (Capel et al. 1998; Carrión et al. 2001, 2007; Nachasova et al. 2007; Yanes et al. 2011). Esta tendencia, por otra parte, se ha revelado a partir de estudios isotópicos sobre restos humanos de tumbas megalíticas del sureste pero atribuyéndose a modificaciones en las estrategias agrarias (Díaz-Zorita et al. 2019).

Además la forma de las curvas de la evolución de las medias a lo largo de las fases muestra que las condiciones medioambientales, en combinación posiblemente con otros factores, influyeron de forma parecida en los valores $\delta(\%)$ de ambos isótopos, aunque más apreciables en el caso de los valores $\delta^{13} \mathrm{C}$.

Para la única muestra del Cobre Antiguo el valor en $\delta^{15} \mathrm{~N}$ es de 10,14 , mientras el valor en $\delta^{13} \mathrm{C}$ es de $-19,09$. En ambos, con todas las muestras y también con la única datada, los valores son más elevados que las medias de todo el conjunto de muestras. Ello podría implicar diferencias en consumo, con un aporte mayor de proteínas animales. Los valores del carbono nos sugieren que un factor a considerar es el componente ambiental, indicativo de un clima relativamente seco en el Cobre Antiguo, previo a un aumento de la humedad en torno al $2800 \mathrm{cal}$ AC. Las condiciones áridas 

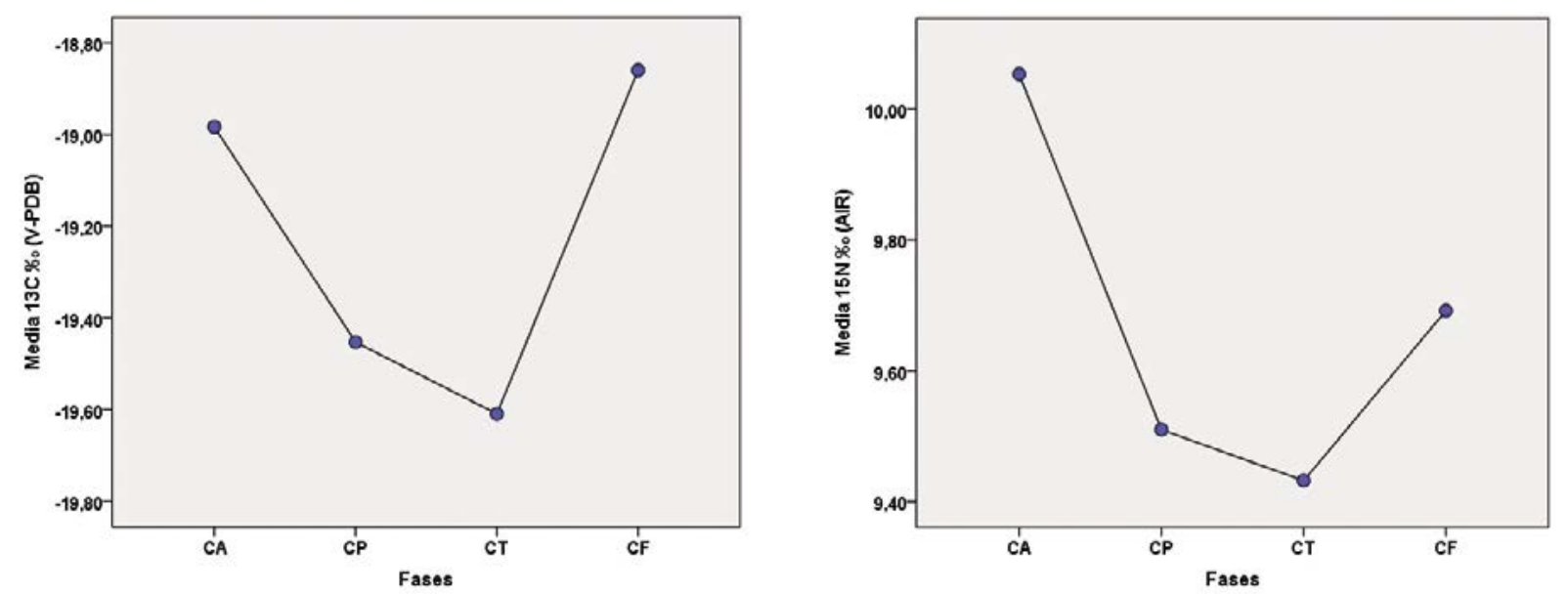

Fig. 4. Medias de $\delta^{15} \mathrm{~N}$ y $\delta^{13} \mathrm{C}$ por fases considerando todas las muestras estudiadas de la necrópolis de Los Millares.

serían la continuación de un proceso que se habría iniciado entre el 4000/3800 a. C. en amplias zonas del Mediterráneo (Gasse y Van Campo 1994; Magny et al. 2009; Mayewski et al. 2004; Giraudi et al. 2011, 2013; Pérez-Obiol et al. 2011; Jaouadi et al. 2016).

La situación cambia durante el periodo siguiente o Cobre Pleno, fechado en el segundo cuarto del III milenio a. C. Considerando solo las cuatro muestras datadas la media en los valores $\delta^{15} \mathrm{~N}$ es de $\pm 9,63$ con una desviación típica de 0,22 , mientras la media en $\delta^{13} \mathrm{C}$ es de $-19,19$ con una desviación típica de 0,15 .

Este posible incremento de la humedad, como sucede en varios yacimientos donde se ha constatado con otros tipos de análisis (Carrión et al. 2001, 2007; Nachasova et al. 2007; Yanes et al. 2011, 2013; Rodríguez 2012), se acentúa en el periodo siguiente (Cobre Tardío), en los siglos centrales del III milenio a. C. Ello también coincide con los valores $\delta^{13} \mathrm{C}$ medios más negativos del conjunto de muestras atribuidas a este periodo. De hecho, si nos circunscribimos a las 4 muestras datadas correspondientes a este último periodo, la media en los valores $\delta^{15} \mathrm{~N}$ es de $\pm 9,29$ con una desviación típica de 0,99 , mientras la media en $\delta^{13} \mathrm{C}$ es de $-19,22$ con una desviación típica de 0,27.

En el Cobre Final, si nos centramos en las 4 muestras datadas, la media en los valores $\delta^{15} \mathrm{~N}$ es de $\pm 9,86$ con una desviación típica de 0,96 , mientras la media en $\delta^{13} \mathrm{C}$ es de $-18,69$ con una desviación típica de 0,09 . Este cambio hacia valores menos negativos implica un incremento de la eficiencia al uso del agua de las plantas, es decir, mayor aridez. Por tanto, los datos isotópicos del colágeno indican el fuerte cambio con respecto al período anterior, apreciado ya en el conjunto de las muestras disponibles. Este cambio coincide con el proceso hacia una mayor aridez en la transición hacia el II milenio a. C. visible no solo en Los Millares (Rodríguez y Vernet 1991; Capel et al. 1998) sino en otros yacimientos (Carrión et al. 2001, 2007; Nachasova et al. 2007; Yanes et al. 2011), aunque, tanto para los cambios en los valores isotópicos (Díaz-Zorita et al. 2019), como para la caída en la cobertura vegetal, se hayan dado también interpretaciones que enfatizan la acción humana (Rodríguez 1992, 2018; Fuentes et al. 2007). Por otro lado, se mantiene la tendencia a la mayor variabilidad en los valores del nitrógeno apreciada en el periodo anterior. Ello nos permite pensar en una consolidación de las diferencias en dieta cárnica, dado que, además como hemos indicado, no parece haber diferencias por edad, aunque ahora sean más frecuentes los enterramientos infantiles (Mathers 1984; Peña 2011). Estos llegan a ser habituales en contextos funerarios peninsulares, sobre todo de la segunda mitad del III milenio a. C. (Waterman y Thomas 2011; Beck 2016; Cámara et al. 2016), pero recordemos que, respecto a los valores isotópicos en nitrógeno y carbono, no muestran sustanciales diferencias con los adultos en Los Millares.

\subsection{Discusión}

Los valores isotópicos del colágeno en la mayor parte de las muestras analizadas son compatibles con una dieta basada en recursos terrestres (Fig. 5). Los valores que se salen del rango principal con una correlación positiva de valores altos en $\delta^{15} \mathrm{~N}$ y $\delta^{13} \mathrm{C}$ son compatibles con la inclusión en la dieta de una proporción de peces, aves marinas y/o predadores marinos altos en la cadena trófica. Este consumo se había constatado ya con los análisis faunísticos en Los Millares (alcatraz, 


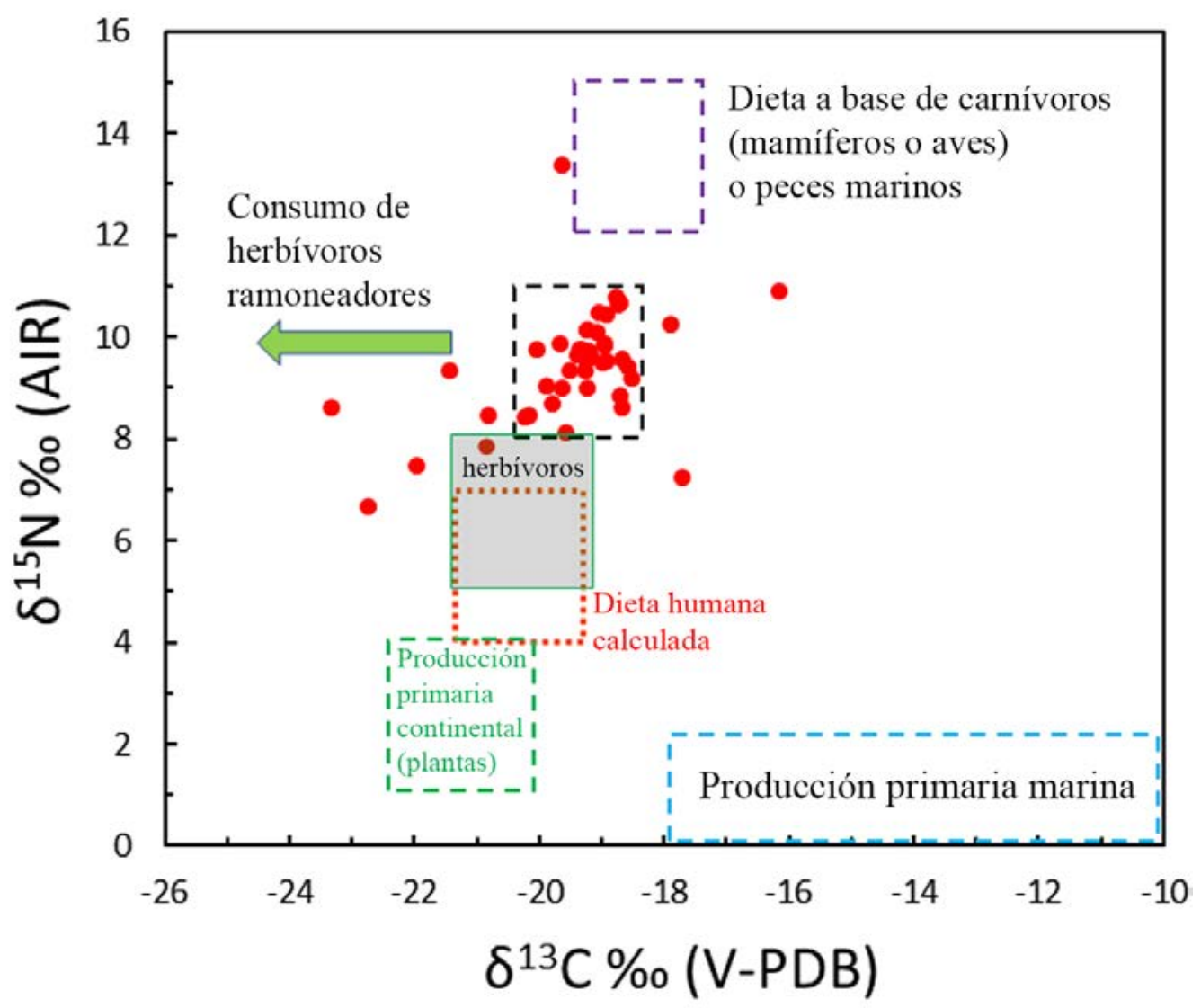

Fig. 5. Representación de los valores obtenidos en este estudio para los restos humanos de la necrópolis de Los Millares respecto a los valores producidos por diferentes grupos de plantas y animales. El recuadro relleno indica el valor teórico de los herbívoros. La mayoría de las muestras de los Millares se encuadran en el área delimitada por trazado discontinuo, a partir de la cual hemos calculado su dieta teórica (cuadrado delimitado con puntos). Como puede observarse, la mayor parte de los individuos tendría una dieta basada en el consumo de herbívoros junto con plantas C3 (gráfico en color en la versión electrónica).

Morus bassanus) (Peters y Driesh 1990). Por el contrario, los casos situados en una correlación negativa (los valores menos negativos en $\delta^{13} \mathrm{C}$, pero relativamente bajos en $\delta^{15} \mathrm{~N}$ ) son compatibles con un consumo de algas marinas y seagrass (ambas con valores relativamente bajos en la relación $\mathrm{C} / \mathrm{N}$, es decir, relativamente ricas en proteínas), o más probablemente moluscos y crustáceos, todos caracterizados por valores menos negativos de $\delta^{13} \mathrm{C}$ (Duarte et al. 2018). Este sería el caso de la muestra 76-1-Mill-48-12. El consumo de moluscos como lapas y bígaros (Patellidae, Phorcus turbinatus) también se constata en el análisis arqueozoológico (Peters y Driesch 1990). En este contexto es importante señalar que la costa está actualmente a solo $18 \mathrm{~km}$ y en la Edad del Cobre pudo estar a una menor distancia, ya que el estuario del río Andarax se ha rellenado de sedimentos debido a la acción antrópica en época histórica (Arteaga y Hoffmann 1999).
Las muestras con valores menores de +9 en $\delta^{15} \mathrm{~N}$ y más negativas en $\delta^{13} \mathrm{C}$ serían compatibles con una dieta en la que habían predominado proteínas de origen vegetal, en algunos casos de productos agrícolas abonados con residuos ganaderos. También los herbívoros que ramoneen árboles, con raíces más profundas, y menor estrés hídrico, presentan valores más negativos en $\delta^{13} \mathrm{C}$, por lo que incluso una dieta esencialmente carnívora podría ser compatible con estos valores menos negativos. Por otra parte, debe indicarse que estos valores más negativos en $\delta^{13} \mathrm{C}$ pertenecen a individuos del periodo 2 (momentos avanzados del Cobre Pleno) o, en mayor número, del período 3 (Cobre Tardío), que corresponderían a una fase más húmeda, constatada en otros análisis paleoambientales sobre zonas cercanas (Carrión et al. 2001; Nachasova et al. 2007; Yanes et al. 2011, 2013) y deducible de los resultados de otros análisis isotópicos sobre restos humanos de sepulturas 
megalíticas del sureste (Díaz-Zorita et al. 2019). Uno de los individuos es particularmente reseñable, ya que representa un eslabón por encima en la cadena trófica, con valores relativamente altos en $\delta^{15} \mathrm{~N}$ y valores dentro de la normalidad en $\delta^{13} \mathrm{C}$. Este caso podría explicarse por una dieta basada en carnívoros, ya sean mamíferos o aves silvestres (terrestres), como hemos indicado ya en el comentario general. Al tratarse de una mujer, se podría pensar en una procedencia de otro asentamiento, para evitar efectos endogámicos dentro de una dinámica patrilocal, aspecto que ya se ha referido en yacimientos de la Edad del Bronce de la Alta Andalucía como el Cerro del Alcázar (Baeza, Jaén) (Molina et al. 2019). Hay que señalar, sin embargo, la necesidad de otros estudios que prueben de manera más fehaciente una movilidad sin duda existente también en el Calcolítico (Díaz-Zorita et al. 2018).

Los valores $\delta^{13} \mathrm{C}$ calculados para la producción primaria terrestre $(-22,5$ a $-20,5 \%$ vs V-PDB) están en el rango de las gramíneas (cereales) del sur de la península ibérica (Mora 2017), siendo menos negativos que los cereales (entre -23 y -26) del noroeste (Mora et al. 2018) con un clima muy húmedo $\mathrm{y}$, por tanto, menor WUE y más discriminación isotópica que en los ambientes áridos (Farquhar y Richards 1984). Por otra parte, considerando áreas áridas del este de la península donde los cereales irrigados estaban comprendidos entre -23 y $-25 \%$, en el mismo rango que los pinos con mayor capacidad radicular y menos eficiencia en el aprovechamiento del agua (WUE), se ha calculado que las gramíneas en la Edad del Bronce pudieron ser sobre un $3 \%$ menos negativas (debido a una mayor $W U E$ ), estando comprendidas entre -20 y -22 (Mora et al. 2016). Ello estaría en el rango que se deduce a partir de los valores de los humanos de Los Millares. Cabe suponer que el área de Los Millares, en general, durante parte de su ocupación y como ocurre en la actualidad, tenía un clima más árido que el resto de la península ibérica y similar a algunas áreas del este peninsular. Se observa que los valores menos negativos corresponden al Cobre Final lo que se ha relacionado con un incremento de la aridez y, por tanto, un aumento de la eficiencia en el aprovechamiento del agua por parte de la cobertera vegetal, provocando, como se han indicado anteriormente, una menor discriminación isotópica del ${ }^{13} \mathrm{C}$. La muestra de la tumba 33, correspondiente al Bronce Final, nos marca un clima más árido aún, con un valor $\delta^{15} \mathrm{~N}$ de 10,91 y $\delta^{13} \mathrm{C}$ de $-16,18$. Si en las primeras fases del Bronce Final se produjo un aumento considerable de la humedad (Burakov et al. 2005; López-Sáez et al. 2014), es también posible que en torno al siglo IX a. C. el clima sufriera un nuevo deterioro (Jaouadi et al. 2016). Hay que tener en cuenta que la fecha proporcionada por nuestra muestra se sitúa antes de la transición al Subat- lántico y aunque, al menos en áreas más septentrionales, se ha planteado que este no solo implica condiciones más frías sino también más húmedas entre 850 y 750 a. C. (López-Sáez et al. 2009), la disminución de precipitaciones llega hasta el 600/500 a. C. cuando se asientan las condiciones frías, como se ha indicado para otras áreas (Gaigalas 2004; Van Mourik y Slotboom 2018).

En general, hombres y mujeres no presentan diferencias en sus valores, si bien para las mujeres se observa una mayor dispersión, posiblemente relacionada con la necesidad de recurrir a recursos más dispares, como lo indica la dieta más rica en proteínas vegetales o la más rica en proteína animales. Los valores también pueden reflejar que algunas mujeres vinieran de otros poblados, un indicio de exogamia como ya hemos indicado.

Los adultos, en especial los adultos maduros, presentan en general valores altos en la cadena trófica, indicando un predominio de proteínas de origen animal, como se ha referido en otros estudios isotópicos sobre restos humanos del Calcolítico del sureste (Waterman et al. 2017; Díaz-Zorita et al. 2019). Solo los dos adultos maduros, que podrían tener un origen foráneo, presentan valores $\delta^{13} \mathrm{C}$ menos negativos, típicos de una dieta basada en una productividad primaria marina, ya sea debido al consumo de moluscos o algas, en un caso, o al consumo de organismos (peces, mamíferos marinos o aves marinas) en el otro. Recientes estudios han señalado la variabilidad en la dieta de la Prehistoria Reciente, incluyendo productos marinos ocasionales, mostrando como, sin llegar a los hábitos epipaleolíticos, en la fachada mediterránea de la península ibérica se mantuvo esporádicamente el consumo de recursos marinos (Salazar-García et al. 2018). Es importante recordar que las variables ambientales influyen también en los valores obtenidos. Ello explica, en parte, la situación de la muestra correspondiente al enterramiento del Bronce Final. Finalmente, los individuos infantiles parecen tener una dieta más cuidada y centrada en la media. Solo algunos individuos adultos y jóvenes parecen incluir más proteínas vegetales en su dieta.

\section{VALORACIÓN FINAL}

Aunque no es el objetivo principal de este artículo, hay que señalar que las dataciones obtenidas muestran, de forma más clara que en otros estudios (Aranda y Lozano 2014; Lozano y Aranda 2017), que los tholoi están presentes en el sureste ya en la transición entre el IV y el III milenios a. C., en consonancia con lo propuesto para otras zonas de la península ibérica (Boaventura y Mataloto 2013). 
La menor armonización entre los valores $\delta^{15} \mathrm{~N}$ y $\delta^{13} \mathrm{C}$ en algunas de las muestras en cada uno de los periodos podría sugerir diferencias en consumo, aunque los valores isotópicos están muy influidos por las condiciones ambientales. Si estamos en lo cierto respecto a la influencia del clima, dentro de un contexto general relativamente seco, habría que pensar que la aridez disminuye a principios del III milenio a. C. Esto es apreciable tanto en los datos de Los Castillejos de las Peñas de los Gitanos, en las Sierras Subbéticas granadinas (Nachasova et al. 2007; Yanes et al. 2011; Rodríguez 2018) como en el estudio antracológico de Los Millares (Rodríguez y Vernet 1991). Por el contrario, a fines de la ocupación de Los Millares, desde el Cobre Tardío y muy especialmente en el Cobre Final, empiezan a desarrollarse las condiciones de incremento de la aridez que caracterizarán también el II milenio a. C. en todo el sur de la península ibérica (Carrión et al. 2001, 2007; Nachasova et al. 2007; Yanes et al. 2011), aunque, a veces, se tienda a acentuar la acción humana en procesos como el cambio en las especies arbóreas (Rodríguez 1992, 1996, 2018; Rodríguez et al. 1996; Fuentes et al. 2005). Hay que considerar que el inicio del evento árido 4.2 se suele situar en los momentos finales de Los Millares entre el 2400 y el 2100 cal AC, como se ha constatado en distintas áreas del Mediterráneo (Drysdale et al. 2006; Migowski et al. 2006; Magny et al. 2009; Ruan et al. 2016), donde también se ha demostrado que las afecta de manera variable (Lillios et al. 2016; Zanchetta et al. 2016).

El número de individuos en los que se ha podido determinar la edad es aún bajo, pero llama la atención la ausencia de diferencias entre los individuos infantiles y los adultos en los valores $\delta^{15} \mathrm{~N}$. Esto contrasta con lo conocido para los yacimientos de la Edad del Bronce del sureste de la península ibérica (Molina et al. 2016, 2019) y para yacimientos calcolíticos de áreas cercanas (Beck et al. 2018).

Frente a lo sugerido para el yacimiento de Marroquíes (Jaén) (Beck et al. 2018), los datos hasta ahora disponibles para Los Millares no nos permiten plantear diferencias de acceso a los recursos cárnicos por parte de los individuos inhumados en las diferentes tumbas, aunque esas diferencias de acceso sí aparezcan en la distribución de los restos faunísticos de las zonas de hábitat (Navas et al. 2008). Un aspecto que se debe considerar en relación con estos resultados es que las tumbas que se nos ha permitido muestrear, en general, ofrecían un ajuar escaso. Los valores de la tumba 57 son relativamente altos en $\delta^{15} \mathrm{~N}$ pero son superados, $\mathrm{p}$. ej., por los de la tumba 74, cuyo ajuar es también relativamente rico, sobre todo si tenemos en cuenta el escaso número de cadáveres identificado, pero también por los valores de los individuos muestreados correspondientes a las tumbas 51 y 52 , de escaso ajuar. En nuestra opinión son las diferencias temporales y la influencia del ambiente las causas que enmascaran las posibles diferencias sociales en dieta. De hecho, el aspecto más relevante de las diferencias en consumo es la posibilidad de que algunos individuos recurrieran a proteínas de origen marino. Este aspecto se había constatado también en otros estudios para la fachada mediterránea de la península ibérica (Salazar-García et al. 2018), pero no se había planteado en los estudios previos sobre muestras calcolíticas del sureste (Waterman et al. 2017; Díaz-Zorita et al. 2019).

\section{AGRADECIMIENTOS}

A Carmen Cacho, Eduardo Galán y Ruth Maicas por su autorización para la toma de muestras en el Museo Arqueológico Nacional y su ayuda durante la misma y a Manuel Ramos por la realizada en el Museo Arqueológico de Almería.

\section{BIBLIOGRAFÍA}

Afonso, J. A.; Cámara, J. A.; Martínez, G. y Molina González, F. 2011: "Objetos en materias primas exóticas y estructura jerárquica de las tumbas de la necrópolis de Los Millares (Santa Fe de Mondújar, Almería, España”. En D. W. Wheatley, C. Scarre y L. García Sanjuán (eds.): Explorando el tiempo y la materia en los monumentos prehistóricos: cronología absoluta y rocas raras en los megalitos europeos. Menga Extra 1, Junta de Andalucía. Sevilla: 295-334.

Almagro Basch, M. y Arribas, A. 1963: El poblado y la necrópolis megalíticos de Los Millares (Santa Fe de Mondújar, Almeria). Bibliotheca Praehistorica Hispana 3. CSIC. Madrid.

Aranda, G. y Lozano Medina, A. 2014: "The chronology of megalithic funerary practices: a Bayesian approach to Grave 11 at El Barranquete necropolis (Almería, Spain)". Journal of Archaeological Science 50: 369-382. https://doi.org/10.1016/j.jas.2014.08.005

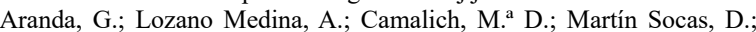
Rodríguez Santos, F. J.; Trujillo, A... y Clop, X. 2017a: "La cronología radiocarbónica de las primeras manifestaciones en el sureste de la Península Ibérica: las necrópolis de Las Churuletas, La Atalaya y Llano del Jautón (Purchena, Almería)". Trabajos de Prehistoria 74 (2): 257-277. https://doi.org/10.3989/tp.2017.12194

Aranda, G.; Lozano Medina, A.; Díaz Zorita, M.; Sánchez Romero, M. y Escudero, J. 2017b: "Cultural continuity and social resistance: the chronology of megalithic funerary practices in Southern Iberia". European Journal of Archaeology 21 (2): 192-216. https://doi.org/10.1017/eaa.2017.42

Arribas, A.; Molina, F.; Carrión, F.; Contreras, F.; Martínez, G.; Ramos, A... y Martínez, J. 1987: "Informe preliminar de los resultados obtenidos durante la VI campaña de excavaciones en el poblado de Los Millares (Santa Fe de Mondújar, Almería). 1985". Anuario Arqueológico de Andalucía 1985. II. Actividades Sistemáticas. Junta de Andalucía. Sevilla: 245-262.

Arribas, A.; Molina González, F.; Sáez, L.; Torre, F. de la; Aguayo, P. y Nájera, T. 1979: "Excavaciones en Los Millares (Santa Fe, Almería). Campañas de 1978 a 1979". Cuadernos de Prehistoria de la Universidad de Granada 4: 61-110.

Arteaga, O. y Hoffmann, G. 1999: "Dialéctica del proceso natural y sociohistórico en las costas mediterráneas de Andalucía". Revista Atlántica-Mediterránea de Prehistoria y Arqueología Social 2: 13121. 
Beck, J. 2016: "Part of the family: age, identity and burial in Copper Age Iberia". En A. Osterholtz (ed.): Theoretical approaches to analysis and interpretation of commingled human remains. Springer International. Cham.: 47-73.

Beck, J.; Díaz-Zorita, M.; Bocherens, H. y Díaz-del-Río, P. 2018: "Feeding a third millennium BC mega-site: Bioarchaeological analyses of palaeodiet and dental disease at Marroquíes (Jaén, Spain)". Journal of Anthropological Archaeology 52: 23-43.

https://doi.org/10.1016/j.jaa.2018.07.001

Blance, B. 1971: Die anfange der metallurgie auf der Iberischen Halbinsel. Studien zu den Anfängen der Metallurgie, 4. Römisch-Gemanisches Zent. Berlin.

Boaventura, R. y Mataloto, R. 2013: "Entre mortos e vivos: nótulas acerca da cronologia absoluta do Megalitismo do Sul de Portugal". Revista Portuguesa de Arqueologia 16: 81-101.

Bocherens, H.; Billiou, D.; Patou-Mathis, M.; Bonjean, D.; Otte, M. y Mariotti, A. 1997: "Paleobiological implications of the isotopic signature $\left({ }^{13} \mathrm{C},{ }^{15} \mathrm{~N}\right)$ of fossil mammal collagen in Scladina cave (Sclayn, Belgium)". Quaternary Research 48 (3): 370-380. https://doi.org/10.1006/qres.1997.1927

Burakov, K. S.; Nachasova, I. E.; Nájera, T.; Molina González, F. y Cámara, J. A. 2005: "Geomagnetic intensity in Spain in the second millennium BC". Izvestiya Physics of the Solid Earth 41 (8): 622-633.

Calvín Velasco, M. ${ }^{\mathrm{a}}$ E. 2014: "Estudio, análisis y valoración social de la necrópolis calcolítica de Los Millares (Santa Fe de Mondújar, Almería)". Arqueología y Territorio 11: 1-13.

Cámara, J. A.; Afonso, J. A. y Molina González, F. 2016: "La ocupación de las Peñas de los Gitanos (Montefrío, Granada) desde el Neolítico al mundo romano. Asentamiento y ritual funerario". En R. Pedregosa (ed.): Arqueología e historia de un paisaje singular: La Peña de los Gitanos, Montefrio (Granada). Ayuntamiento de Montefrío. Granada: $17-121$.

Cámara, J. A.; Alcaraz, F. M.; Molina González, F.; Montufo, A. M. y Spanedda, L. 2014: "Monumentality, Visibility and Routes Control in Southeastern Iberian Megalithic Sites". En B. Schulz Paulsson y B. Gaydarska (eds.): Neolithic and Copper Age monuments: emergence, function and the social construction of the landscape. British Archaeological Reports International Series 2625. Archaeopress. Oxford: 89-106.

Cámara, J. A. y Molina González, F. 2006: "Selection of data, determinism and scientific relevante in interpretations of social development in the Late Prehistory of the Iberian Southeast". En P. Díaz-del-Río y L. García Sanjuán (eds.): Social inequality in Iberian Late Prehistory. British Archaeological Reports, International Series 1525, Archaeopress. Oxford: 21-35.

Capel, J.; Reyes, A.; Delgado, A.; Núñez, R. y Molina, F. 1998: "Paleoclimatic identification based on an Isotope study of travertine from the Copper Age site at Los Millares, South-eastern Spain". Archaeometry 40 (1): 177-185. https://doi.org/10.1111/j.1475-4754.1998.tb00832.x

Carrión, J. S.; Munuera, M.; Dupré, M. y Andrade, A. 2001: “Abrupt vegetation changes in the Segura Mountains of southern Spain throughout the Holocene". Journal of Ecology 89: 783-797. https://doi.org/10.1046/j.0022-0477.2001.00601.x

Carrión, J. S.; Fuentes, N.; González-Sampériz, P.; Sánchez, L.; Finlayson, J. C.; Fernández, S. y Andrade, A. 2007: "Holocene environmental change in a montane region of southern Europe with a long history of human settlement". Quaternary Science Reviews 26: 1455 1475. https://doi.org/10.1016/j.quascirev.2007.03.013

Castro Curel, Z. 1988: "Peines prehistóricos peninsulares". Trabajos de Prehistoria 45 (1): 243-258.

Chapman, R. W. 1978: "The evidence for prehistoric water control in south-east Spain". Journal of Arid Environment 1: 261-274. https://doi.org/10.1111/j.1475-4754.1998.tb00832.x

Chapman, R. W. 1981: "Los Millares y la cronología relativa de la Edad del Cobre en el Sudeste de España". Cuadernos de Prehistoria de la Universidad de Granada 6: 75-89.

Chapman, R. W. 1990: Emerging complexity. The later prehistory of south-east Spain, Iberia and the West Mediterranean. Cambridge University Press. Cambridge.
Díaz-Guardamino, M.; García Sanjuán, L. y Wheatley, D. W. 2015: "The lives of prehistoric monuments in Europe: an introduction". En M. Díaz-Guardamino, L. García Sanjuán y D. W. Wheatley (eds.): The lives of prehistoric monuments in Iron Age, Roman, and Medieval Europe. Oxford University Press. Oxford: 3-17.

Díaz-Zorita, M. 2013: The Copper Age in South-West Spain: a bioarchaeological approach to prehistoric social organization. Durham theses, Durham University. Available at Durham E-Theses Online: http://etheses.dur.ac.uk/9470/

Díaz-Zorita, M.; Beck, J.; Bocherens, H. y Díaz-del-Río, P. 2018: "Isotopic evidence for mobility at large-scale human aggregations in Copper Age Iberia: the mega-site of Marroquíes". Antiquity 92 (364): 991-1007. https://doi.org/10.15184/aqy.2018.33

Díaz-Zorita, M.; Knudson, K. J.; Escudero, J.; Bocherens, H. y García, L. 2017: "Mobility patterns and paleodietary insights into humans and cattle at the Copper Age Mega-site of Valencina (Seville, Spain) through $\delta^{18} \mathrm{O}$ and $\delta^{13} \mathrm{C}$ isotope analyses". Menga: Revista de prehistoria de Andalucía 8: 53-68.

Díaz-Zorita, M.; Prevedorou, E. A.; Buikstra, J.; Knudson, K. J.; Gordon, G. y Anbar, A. 2011: "Movilidad y paleodieta en la comunidad argárica de Gatas: análisis de ${ }^{87} \mathrm{Sr} /{ }^{86} \mathrm{Sr},{ }^{18} \mathrm{O}$ y ${ }^{13} \mathrm{C}$ ". En M. Sánchez (ed.): Memorial Luis Siret. Primer Congreso de Prehistoria de Andalucía. La tutela del patrimonio prehistórico (Antequera 2010): 603-606. Sevilla.

Díaz-Zorita, M.; Waterman, A. J. y Peate, D. W. 2014: "La movilidad de las poblaciones de la Edad del Cobre en el Suroeste de España a través de los análisis de isótopos estables de ${ }^{87} \mathrm{Sr} /{ }^{86} \mathrm{Sr}$ ". En E. García (ed.): Movilidad, contacto y cambio. II Congreso de Prehistoria de Andalucía (Antequera 2012): 111-120. Antequera.

Díaz-Zorita, M.; Aranda, G.; Bocherens, H.; Escudero, J.; Sánchez, M.; Lozano, Á... y Milesi, L. 2019: "Multi-isotopic diet analysis of south-eastern Iberian megalithic populations: the cemeteries of El Barranquete and Panoría". Archaeological and Anthropological Sciences 11 (8): 3681-3698. https://doi.org/10.1007/s12520-0180769-5

Drysdale, R.; Zanchetta, G.; Hellstrom, J.; Maas, R.; Fallick, A.; Pickett, M... y Piccini, L. 2006: "Late Holocene drought responsible for the collapse of Old World civilizations is recorded in an Italian cave flowstone". Geology 34 (2): 101-104. https://doi.org/10.1130/G22103.1

Duarte, C. M.; Delgado-Huertas, A.; Anton, A.; Carrillo-de-Albornoz, P.; López-Sandoval, D. C.; Agustí, S.... y Garcias-Bonet, N. 2018: "Stable Isotope $\left(\delta^{13} \mathrm{C}, \delta^{15} \mathrm{~N}, \delta^{18} \mathrm{O}, \delta \mathrm{D}\right)$ composition and nutrient concentration of Red Sea primary producers". Frontiers in Marine Science 5. https://doi.org/10.3389/fmars.2018.00298

Escoriza Mateu, T. 1991-1992: "La formación social de los Millares y las "producciones simbólicas"'. Cuadernos de Prehistoria y Arqueología de la Universidad de Granada 16-17: 135-165.

Esparza, A.; Velasco, J.; Palomo, S.; Delibes, G.; Arroyo, E. y SalazarGarcía, D. C. 2017: "Familiar kinship? Palaeogenetic and isotopic evidence from a triple burial of the Cogotas I Bronze Age archaeological culture (Soria, Spain)". Oxford Journal of Archaeology 36 (3): 223-242. https://doi.org/10.1111/ojoa.12113

Farquhar, G. y Richards, R. 1984: "Isotopic composition of plant carbon correlates with water-use efficiency of wheat genotypes". Functional Plant Biology 11: 539-552. https://doi.org/10.1071/PP9840539

Fernández-López de Pablo, J.; Salazar-García, D. C.; Subirà, M. E.; Roca, C.; Gómez-Puche, M.; Richards, M. P. y Esquembre, A. 2013: "Late Mesolithic burials at Casa Corona (Villena, Spain): direct radiocarbon and paleodietary evidence of the last forager populations in Eastern Iberia". Journal of Archaeological Science 40: 671-680. http://dx.doi.org/10.1016/j.jas.2012.09.005

Fernández Martínez, V. 2015. Arqueo-Estadística: Métodos cuantitativos en Arqueología. Alianza Editorial. Madrid.

Fontanals-Coll, M.; Subirà, M. E.; Díaz-Zorita, M. y Gibaja, J. F. 2017: "First insight into the Neolithic subsistence economy in the north-east Iberian Peninsula: paleodietary reconstruction through stable isotopes". American Journal of Physical Anthropology 162: 36-50. https://doi.org/10.1002/ajpa.23083

Fontanals-Coll, M.; Subirà, M. E.; Marín-Moratalla, N.; Ruiz, J. y Gibaja, J. F. 2014: "From Sado Valley to Europe: Mesolithic dietary 
practices through different geographic distributions". Journal of Archaeological Science 50: 539-550. http://dx.doi.org/10.1016/j.jas.2014.07.028

Fuentes, N.; Carrión, J. S.; Fernández, S.; Nocete, F.; Lizcano, R. y Pérez, C. 2007: “Análisis polínico de los yacimientos arqueológicos Cerro del Alcázar de Baeza y Las Eras del Alcázar de Úbeda (Jaén)”. Anales de Biología 29, 85-93.

Fuentes, N.; García, M. S.; González, P.; Fernández, S.; Carrión, J. S.; López Campuzano, M. y Medina, J. 2005: "Degradación ecológica y cambio cultural durante los últimos cuatro mil años en el sureste ibérico semiárido". Anales de Biología 27: 69-84.

Fuller, B. T.; Márquez-Grant, N. y Richards, M. P. 2010: "Investigation of diachronic dietary patterns on the islands of Ibiza and Formentera, Spain: Evidence from carbon and nitrogen stable isotope ratio analysis". American Journal of Physical Anthropology 143 (4): 512522. http://dx.doi.org/10.1002/ajpa.21334

Gaigalas, A. 2004: "Environmental study of the Bronze-Iron Age transition period of eastern Europe". En E. M. Scott, A. Y. Alekseev y G. Zaitseva (eds.): Impact of the environment on human migration in Eurasia. Kluwer Academic. Dordrecht: 243-254.

García-Guixé, E.; Subirà, M. E. y Richards, M. P. 2006: "Paleodiets of humans and fauna from the Spanish Mesolithic site of El Collado". Current Anthropology 47 (3): 549-556.

http://dx.doi.org/10.1086/504170

Gasse, F. y Van Campo, E. 1994: "Abrupt post-glacial climate events in West Asia and North Africa monsoon domains". Earth and Planetary Science Letters 126 (4): 435-456. https://doi.org/10.1016/0012-821X(94)90123-6

Gibaja, J. F.; Subirà, M. E.; Terradas, X.; Santos, F. J.; Agulló, L.; Gómez Martínez, I.... y Fernández-López de Pablo, J. 2015: "The emergence of Mesolithic cemeteries in SW Europe: insights from the El Collado (Oliva, Valencia, Spain) radiocarbon record". Plos One 10 (1). http://dx.doi.org/10.1371/journal.pone.0115505

Gilman, A. 1976: "Bronze Age dynamics in southeast Spain". Dialectical Anthropology 1: 307-319.

Giraudi, C.; Magny, M.; Zanchetta, G. y Drysdale, R. N. 2011: "The Holocene climatic evolution of Mediterranean Italy: A review of the continental geological data". The Holocene 21 (1): 105-115. https://doi.org/10.1177/0959683610377529

Giraudi, C.; Mercuri, A. M. y Esu, D. 2013: "Holocene palaeoclimate in the northern Sahara margin (Jefara Plain, northwestern Libya)". The Holocene 23: 339-352. https://doi.org/10.1177/0959683612460787

Haro, M.; Carrión, F. y García González, D. 2006: “Territorio y georrecursos en el cabo de Gata (Níjar, Almería) durante la Edad del Cobre". En G. Martínez, A. Morgado y J. A. Afonso (eds.): Sociedades prehistóricas, recursos abióticos y territorio. Universidad de Granada. Granada: 315-326.

Hernando Gonzalo, A. 1987-1988: "Interpretaciones culturales del Calcolítico del Sureste español. Estudio de sus bases teóricas". Cuadernos de Prehistoria de la Universidad de Granada 12-13: 35-80.

Jaouadi, S.; Lebreton, V.; Bout-Roumazeilles, V.; Siani, G.; Lakhdar, R.; Boussoffara, R.... y Combourieu-Nebout, N. 2016: "Environmental changes, climate and anthropogenic impact in southern-eastern Tunisia during the last 8 kyr". Climate of the Past 12 (6): 1339-1359. https://doi.org/10.5194/cp-2016-25

Leisner, G. y Leisner, V. 1943: Die megalithgräber der Iberischen Halbinsel. Der Süden. Romisch-Germanisch Forschungen 17, 2 vols., W. de Gruyter. Berlin.

Lillios, K. T.; Blanco-Gonzalez, A.; Drake, B. L. y López-Sáez, A. 2016: "Mid-late Holocene climate, demography, and cultural dynamics in Iberia: a multi-proxy approach". Quaternary Science Reviews 135: 138-153. https://doi.org/10.1016/j.quascirev.2016.01.011

López-Sáez, J. A.; Alba, F.; Nájera, T.; Molina González, F.; Pérez Díaz, S. y Sabariego, S. 2014: "Paleoambiente y sociedad en la Edad del Bronce de la Mancha: La Motilla del Azuer". Cuadernos de Prehistoria de la Universidad de Granada 24: 391-422.

López-Sáez, J. A.; Blanco-González, A.; López Merino, L.; Ruiz Zapata, M. B.; Dorado Valiño, M.; Pérez Díaz, S... y Burjachs, F. 2009: "Landscape and climatic changes during the end of the Late Prehistory in the Amblés Valley (Ávila, central Spain), from 1200 to 400 cal BC". Quaternary International 200: 90-101. https://doi.org/10.1016/j.quaint.2008.07.010

Lorrio A. J. 2008: Qurénima. El Bronce Final del Sureste de la Península Ibérica. Bibliotheca Archaeologica Hispana 27-Anejos Lucentum 17. Real Academia de la Historia. Madrid.

Lozano Medina, A. y Aranda 2017: "La temporalidad de las sepulturas megalíticas de tipo tholos del sur de la península ibérica”. Spal 26: 17-32. http://dx.doi.org/10.12795/spal.2017i26.01

Lozano Rodríguez, J. A.; Carrión, F.; Morgado, A.; García González, J. D.; Afonso, J. A.; Martínez, G.... y Cámara, J. A. 2010: "Materias primas, productos líticos y circulación. Informe preliminar del estudio de los ajuares de la necrópolis de Los Millares (Santa Fe de Mondújar, Almería)". En S. Domínguez, J. Ramos, J. M. ${ }^{a}$ Gutiérrez y M. Pérez Rodríguez (eds.): Minerales y rocas en las sociedades de la Prehistoria. Universidad de Cádiz. Cádiz: 285-295.

Maicas Ramos, R. 2005: "Rituales de enterramiento en la cuenca de Vera”. En P. Arias, R. Ontañón y C. García-Moncó (eds.): III Congreso del Neolítico en la Península Ibérica (Santander 2003): 767774. Santander.

Maicas Ramos, R. 2019: "The bone industry of Los Millares. From Luis Siret to present". Cuadernos de Prehistoria y Arqueología de la Universidad de Granada 29: 203-218. https://doi.org/10.30827/CPAG.v29i0.9773

Magny, M.; Vannière, B.; Zanchetta, G.; Fouache, E.; Touchais, G.; Petrika, L... y Arnaud, F. 2009: "Possible complexity of the climatic event around 4300-3800 cal. BP in the central and western Mediterranean". The Holocene 19: 823-833. https://doi.org/10.1177/0959683609337360

Martínez Sánchez, R.M.; Tejedor García, Ú.; López Flores, I; DelgadoHuertas, A.; Granados Torres, A.; Tovar Acedo, L.R. y Clapés Salmoral, R. 2020. "Archaeology, chronology, and age-diet insights of two late fourth millennium cal BC pit graves from central southern Iberia (Córdoba, Spain)". International Journal of Osteoarchaeology. 1-11. https://doi.org/10.1002/oa.2853

Mathers, C. 1984: "Beyond the grave: the context and wider implications of mortuary practice in South-Eastern Spain". En T. F. C. Blagg, R. F. J. Jones y S. J. Keay (eds.): Papers in Iberian Archaeology. British Archaeological Reports International Series 193, BAR Publising. Oxford: 13-46.

Mayewski, P. A.; Rohling, E. E.; Stager, J. C.; Karlén, W.; Maasch, K. A.; Meeker, L. D.... y Steig, E. J. 2004: "Holocene climate variability". Quaternary Research 62: 243-255. https://doi.org/10.1016/j.yqres.2004.07.001

McClure, S. B.; García, O.; Roca, C.; Culleton, B. J. y Kennett, D. J. 2011: "Osteological and pelodietary investigation of burials from Cova de la Pastora, Alicante, Spain". Journal of Archaeological Science 38: 420-428. https://doi.org/10.1016/j.jas.2010.09.023

Mederos Martín, A. 1993: Los Estados Incipientes del sureste de la Península Ibérica. Repercusiones en las cuencas de los rios Aguas, Antas y Almanzora. Almería (4500-1300 a.C./5300-1600 A.C.). Serie Tesis Doctorales. Curso 1993/94. Humanidades y Ciencias Sociales. Universidad de La Laguna. La Laguna. http://riull.ull.es/xmlui/handle/915/10097

Micó Pérez, R. 1991: “Objeto y discurso arqueológico. El Calcolítico del sudeste peninsular". Revista d'Arqueologia de Ponent 1: 51-70.

Migowski, C.; Stein, M.; Prasad, S.; Negendank, J. F. y Agnon, A. 2006: "Holocene climate variability and cultural evolution in the Near East from the Dead Sea sedimentary record". Quaternary Research 66 (3): 421-431. https://doi.org/10.1016/j.yqres.2006.06.010 422

Molina González, F. y Cámara, J. A. 2005: Guía del yacimiento arqueológico Los Millares. Consejería de Cultura, Junta de Andalucía. Sevilla.

Molina González, F.; Cámara, J. A.; Delgado, A.; Jiménez Brobeil, S. A.; Nájera, T.; Riquelme, J. A. y Spaneda, L. 2016: "Problemas cronológicos y análisis de dieta en la Edad del Bronce de los altiplanos granadinos". En H. Bonet (ed.): Del neolitic a l'edat de bronze en el Mediterrani occidental: Estudis en homenatge a Bernat Marti Oliver. Servicio de Investigación Prehistórica, Trabajos Varios 119. Valencia: 451-464.

Molina González, F.; Cámara, J. A.; Capel, J.; Nájera, T. y Sáez, L. 2004: "Los Millares y la periodización de la Prehistoria Reciente del

Trab. Prehist., 77, N. ${ }^{\circ}$ 1, enero-junio 2020, pp. 67-86, ISSN: 0082-5638

https://doi.org/10.3989/tp.2020.12247 
Sudeste". En Simposios de Prehistoria Cueva de Nerja. II. La problemática del Neolítico en Andalucía. III. Las primeras sociedades metalúrgicas en Andalucia. Fundación Cueva de Nerja. Nerja: 142158.

Molina, F.; Nocete, F.; Delgado, A.; Cámara, J. A.; Martínez, R. M..; Jiménez, S. A.... y Carrión, F. 2019: "Diet and environment in Southeastern Iberia during the Bronze Age based on isotope analysis of human remains". Oxford Journal of Archaeology 38 (2): 189-213. https://doi.org/10.1111/ojoa.12164

Montero Ruiz, I. 1994: El origen de la metalurgia en el sudoeste de la Península Ibérica, Instituto de Estudios Almerienses, Almería.

Mora, A. 2017: Irrigación y secano en el Mediterráneo Occidental (IIII milenio a.n.e): un estudio isotópico. Tesis Doctoral, Universidad de Granada. http://hdl.handle.net/10481/48605

Mora, A.; Delgado, A.; Granados, A.; Contreras, F.; Jover, F. J. y López, J. A. 2016: "The isotopic footprint of irrigation in the western Mediterranean basin during the Bronze Age: the settlement of Terlinques, southeast Iberian Peninsula". Vegetation History and Archaeobotany 25 (5): 459-468. https://doi.org/10.1007/s00334-016-0560-4

Mora, A.; Delgado, A.; Granados, A.; Contreras, F.; Pavón, I. y Duque, D. 2018: "Complex agriculture during the second millennium bc: isotope composition of carbon studies $\left(\delta^{13} \mathrm{C}\right)$ in archaeological plants of the settlement Cerro del Castillo de Alange (SW Iberian Peninsula, Spain)”. Vegetation History and Archaeobotany 27 (3): 453-462. https://doi.org/10.1007/s00334-017-0634-y.

Nachasova, I. E.; Burakov, K. S.; Molina González, F. y Cámara, J. A. 2007: "Archaeomagnetic study of ceramics from the Neolithic Los Castillejos multilayer monument (Montefrío, Spain)". Izvestiya. Physics of the Solid Earth 43 (2): 170-176. http://dx.doi.org/10.1134/S1069351307020073

Navas, E.; Esquivel, J. A. y Molina González, F. 2008: "Butchering patterns and spatial distribution of faunal animal remains consumed at the Los Millares Chalcolithic settlement (Santa Fe de Mondújar, Almería, Spain)". Oxford Journal of Archaeology 27: 325-339. https://doi.org/10.1111/j.1468-0092.2008.00311.x

Navas, E.; Molina González, F. y Esquivel, J. A. 2005: "La distribución espacial de los restos faunísticos de Los Millares (Santa Fe de Mondújar, Almería)". Complutum 16: 89-104.

Nehlich, O.; Fuller, B. T.; Márquez-Grant, N. y Richards, M. P. 2012: "Investigation of diachronic dietary patterns on the islands of Ibiza and Formentera, Spain: Evidence from sulfur stable isotope ratio analysis". American Journal of Physical Anthropology 149 (1): 115 124. https://doi.org/10.1002/ajpa.22104

Peña Romo, V. 2011: "Excavando huesos en los museos. El caso de la necrópolis de 'Los Millares"”. En A. González Martín, O. CambraMoo, J. Rascón, M. Campo, M. Robledo, E. Labajo y J. A. Sánchez (eds.): Paleopatología: ciencia multidisciplinar. Congreso Nacional de Paleopatología (Madrid 2009): 73-80. Madrid.

Pérez-Obiol, R.; Jalut, G.; Julià, R.; Pèlachs, A.; Iriarte, M. J.; Otto, T. y Hernández-Beloqui, B. 2011: "Mid-Holocene vegetation and climatic history of the Iberian Peninsula". The Holocene 21: 75-93. https://doi.org/10.1177/0959683610384161

Peters, J. y Driesch, A. von den 1990: "Archäozoologische Untersunchung der Tierreste aus der Kupferzeitlichen Siedlung von Los Millares (prov. Almeria)". En Studien über frühe Tierknochenfunde von der Iberichen Halbinsel 12. München: 49-120.

Reimer, P. J.; Bard, E.; Bayliss, A.; Beck, J. W.; Blackwell, P. G.; Ramsey, C. B... y van der Plicht, J. 2013: "IntCal13 and Marine13 Radiocarbon Age Calibration Curves 0-50.000 years cal BP". Radiocarbon 55 (4): 1869-1887. https://doi.org/10.2458/azu js rc.55-16947

Rodríguez Ariza, M. ${ }^{a}$ O. 1989-1990: "Una aplicación del análisis de correspondencias en la valoración del antracoanálisis de Los Millares". Cuadernos de Prehistoria y Arqueología de la Universidad de Granada 14-15: 81-108.

Rodríguez Ariza, M. ${ }^{\text {a }}$ O. 1992: Las relaciones hombre-vegetación en el sureste de la Península Ibérica durante las Edades del Cobre y Bronce a partir del análisis antracológico de siete yacimientos arqueológicos. Editorial Universidad de Granada. Granada. http://hdl.handle.net/10481/14184

Rodríguez Ariza, M. ${ }^{\text {a }}$ O. 1996: "Análisis antracológicos de yacimientos neolíticos de Andalucía”. Rubricatum 1: 73-83.
Rodríguez Ariza, M. ${ }^{a}$ O. 2012: "Depresión de Baza-Huéscar, Granada". En J. S. Carrión (ed.): Paleoflora y paleovegetación de la Península Ibérica e Islas Baleares: Plioceno-Cuaternario. Universidad de Murcia. Murcia: 898-900.

Rodríguez Ariza, M. ${ }^{a}$ O. 2018: "Mid-Holocene local vegetation dynamics and human impact at Los Castillejos, Andalusia, Spain: evidence from charcoal analysis". Vegetation History and Archaeobotany 27: 843-856. https://doi.org/10.1007/s00334-018-0673-z

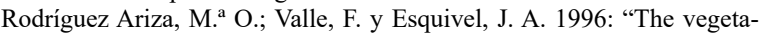
tion from the Guadix-Baza (Granada, Spain) during the Copper and Bronze Ages based on Anthracology". Archeologia e Calcolatori 7: 537-558.

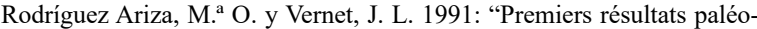
cologiques de l'établissement chalcolithique de Los Millares (Santa Fé de Mondujar, Almería, Espagne) d'après l'analyse anthracologique”. En W. H. Waldren, J. A. Ensenyat y R. C. Kennard (eds.): $2^{\text {nd }}$ Deya Conference of Prehistory. Archaeological techniques, technology and theory (Deià 1988). British Archaeological Reports International Series 573, Tempus Reparatum. Oxford: 1-16.

Ruan, J.; Kherbouche, F.; Genty, D.; Blamart, D.; Cheng, H.; Dewilde, F... y Michelot, J. L. 2016: "Evidence of a prolonged drought ca. $4200 \mathrm{yr}$ BP correlated with prehistoric settlement abandonment from the Gueldaman GLD1 cave, Northern Algeria". Climate of the Past 12 (1): 1-14. https://doi.org/10.5194/cp-12-1-2016

Salazar García, D. C. 2009: "Estudio de la dieta en la población neolítica de Costamar. Resultados preliminares de análisis de isótopos estables de C y N". En E. Flors (ed.): Torre la Sal (Ribera de Cabanes, Castellón). Evolución del paisaje antrópico desde la Prehistoria hasta el Medioevo. Monografies de Prehistòria i Arqueologia Castellonenques 8, Diputació de Castelló. Castelló: 411-420.

Salazar García, D. C. 2011: "Aproximación a la dieta de la población calcolítica de La Vital a través del análisis de isótopos estables del carbono y del nitrógeno sobre restos óseos”. En G. Pérez Jordà, J. Bernabeu, Y. Carrión, O. García Puchol, L. Molina y M. Gómez Puche (eds.): La Vital (Gandia, Valencia). Vida y muerte en la desembocadura del Serpis durante el III y el I milenio a.C. Trabajos Varios del Servicio de Investigación Prehistórica 113. Valencia: 139144.

Salazar García, D. C.; Aura, J. E.; Olària, C. R.; Talamo, S.; Morales, J. V. y Richards, M. P. 2014a: "Isotope evidence for the use of marine resources in the Eastern Iberian Mesolithic". Journal of Archaeological Science, 42: 231-240. http://dx.doi.org/10.1016/j.jas.2013.11.006

Salazar-García, D. C.; Fontanals-Coll, M.; Goude, G. y Subirà, M. E. 2018: "To 'seafood' or not to 'seafood'? An isotopic perspective on dietary preferences at the Mesolithic-Neolithic transition in the Western Mediterranean". Quaternary International 470: 497-510. https://doi.org/10.1073/pnas.1607810113

Salazar García, D. C. y Pinto, V. Silva 2017: "Isótopos en la Prehistoria y Arqueología valencianas". En F. Arasa y C. Mata (eds.): Homenaje a la profesora Carmen Aranegui Gascó. Saguntum Extra 19: 7591.

Salazar García, D. C.; Richards, M. P.; Nehlich, O. y Henry, A. G. 2014b: "Dental calculus is not equivalent to bone collagen for isotope analysis: a comparison between carbon and nitrogen stable isotope analysis of bulk dental calculus, bone and dentine collagen from same individuals from the Medieval site of El Raval (Alicante, Spain)". Journal of Archaeological Science 47: 70-77. http://dx.doi.org/10.1016/j.jas.2014.03.026

Salazar García, D. C.; Romero, A.; García-Borja, P.; Subirà, E. y Richards, M. P. 2016: "A combined dietary approach using isotope and dental buccal-microwear analysis of humans from the Neolithic, Roman and Medieval periods from archaeological site of Tossal de les Basses (Alicante, Spain)". Journal of Archaeological Science, Reports 6: 610-619. http://dx.doi.org/10.1016/j.jasrep.2016.03.002

Schwabedissen, H. y Freundlich, J. 1966: "Köln radiocarbon measurements I". Radiocarbon 8: 239-247. https://doi.org/10.1017/S003382220000014X

Siret, L. 1893: "L'Espagne préhistorique". Revue des Questions Scientifiques 34: 21-78. 
Siret, L. 1913: Questions de chronologie et d'ethnographie ibériques. I. De la fin du quaternaire a la fin du bronce. P. Geuthner. Paris.

Sokal, R. R. y Rohlf, F. J. 2012: Biometry. Freeman and Company. New York.

Van Mourik, J. M. y Slotboom, R. T. 2018: "Palynological reconstruction of the effects of Holocene climatic oscillations and agricultural history on soils and landforms in Luxembourg". En A. M. Kooijman, L. H. Cammeraat y A. C. Seijmonsbergen (eds.): The Luxembourg Gutland Landscape. Springer. Cham: 39-72.

Van Strydonck, M.; Boudin, M.; Ervynck, A.; Orvay, J. y Borms, H. 2005: "Spatial and temporal variation of dietary habits during the prehistory of the Balearic Islands as reflected by ${ }^{14} \mathrm{C}, \delta^{15} \mathrm{~N}$ and $\delta^{13} \mathrm{C}$ analyses on human and animal bones". Mayurqa 30: 523-541.

Venables, W. N. y Ripley, B. D. 2002: Modern Applied Statistics with $S$. Springer Verlag. New York.

Villalba-Mouco, V.; Utrilla, P.; Laborda, R.; Lorenzo, J. I.; Martínez Labarga, C. y Salazar García, D. C. 2018: "Reconstruction of human subsistence and husbandry strategies from the Iberian Early Neolithic: A stable isotope approach". American Journal of Physical Anthropology 2018: 1-15. https://doi.org/10.1002/ajpa.23622

Waterman, A. J.; Beck, J. L.; Thomas, J. T. y Tykot, R. H. 2017: "Stable isotope analysis of human remains from Los Millares cementery (Almería, Spain, c. 3200-2200 cal BC): regional comparisions and dietary variability". Menga 8: 15-27.
Waterman, A. J.; Silva, A. M. y Tykot, R. H., 2014. "Stable isotopic indicators of diet from two Late Prehistoric burial sites in Portugal: an investigation of dietary evidence of social differentiation". Open Journal of Archaeometry 2: 22-27. https://doi.org/10.4081/arc.2014.5258

Waterman, A. J. y Thomas, J. T. 2011: "When the bough breaks: childhood mortality and burial practice in Late Neolithic Atlantic Europe". Oxford Journal of Archaeology 30 (2): 165-183. http://dx.doi.org/10.1111/j.1468-0092.2011.00363.x

Yanes, Y.; Riquelme, J. A.; Cámara, J. A. y Delgado, A. 2013: "Stable isotopes composition of middle to late Holocene land snail shells from the Marroquíes archaeological site (Jaén, Southern Spain). Paleoenvironmental implications". Quaternary International 302: 7787. http://dx.doi.org/10.1016/j.quaint.2012.06.037

Yanes, Y.; Romanek, C. S.; Molina González, F.; Cámara, J. A. y Delgado, A. 2011: "Holocene Paleoenvironment ( 7200-4000 cal BP) of the Los Castillejos Archaeological site (SE Spain) inferred from the stable isotopes of land snail shells". Quaternary International 244: 67-75. http://dx.doi.org/10.1016/j.quaint.2011.04.031

Zanchetta, G.; Regattieri, E.; Isola, I.; Drysdale, R. N.; Bini, M.; Baneschi, I. y Hellstrom, J. C. 2016: "The so-called '4.2 event' in the Central Mediterranean and its climatic teleconnections". Alpine and Mediterranean Quaternary 29 (1): 5-17. 